\title{
Stripes in cuprate superconductors: Excitations and dynamic dichotomy
}

\author{
G. Seibold* \\ Institut für Physik, BTU Cottbus, PBox 101344, 03013 Cottbus, Germany \\ M. Grilli, J. Lorenzana \\ SMC-INFM-CNR and Dipartimento di Fisica, Università di Roma "La Sapienza", P.le Aldo Moro 5, I-00185 Roma, Italy
}

\begin{abstract}
We present a short account of the present experimental situation of stripes in cuprates followed by a review of our present understanding of their ground state and excited state properties. Collective modes, the dynamical structure factor, and the optical conductivity of stripes are computed using the time-dependent Gutzwiller approximation applied to realistic one band and three band Hubbard models, and are found to be in excellent agreement with experiment. On the other hand, experiments like angle-resolved photoemission and scanning tunneling microscopy show the coexistence of stripes at high energies with Fermi liquid quasiparticles at low energies. We show that a phenomenological model going beyond mean-field can reconcile this dynamic dichotomy.
\end{abstract}

Keywords: high-temperature superconductors, stripes, electronic structure, transport properties, charge excitations, magnetic excitations

\section{Introduction}

Since the discovery of high-temperature superconductors (HTSC) by Bednorz and Müller [1] numerous experiments have evidenced the existence of electronic inhomogeneities in these compounds (cf. e.g. Ref. [2]). While early on these inhomogeneities where believed to be predominantly chemically driven, e.g. due to material imperfections like disorder induced by the dopant ions, it was subsequently realized that the strongly correlated character of the cuprate superconductors and thus the electronic subsystem itself can favor the formation of inhomogeneities on the nano-scale. According to the analyis by the Rome group [3] the inherent reduction of the quasiparticle kinetic energy together with a short range attractive (e.g. electron-lattice) interaction can induce an instability towards phase separation since competing nesting instabilities are suppressed due to a residual repulsion of the quasiparticles at large momenta. On the other hand, the long-range repulsive Coulomb interaction will spoil the associated zero-momentum instability in the charge sector and instead shift the wave-

\footnotetext{
${ }^{*}$ corresponding author

Email address: goetz@physik.tu-cottbus.de (G. Seibold)
}

vector of the ordering transition to finite values. This socalled frustrated phase separation mechanism [4] thus results in an incommensurate charge ordering (CO).

An alternative approach is based on Hartree-Fock (HF) investigations of Hubbard (and $\mathrm{tJ}$ )-type hamiltonians $[5,6,7,8]$ which have revealed solutions with a combined charge- and spin-density wave. In contrast to the frustrated phase separation mechanism the charge order in this case is driven by a spin-density wave instability which via the coupling between longitudinal spin and charge degrees of freedom results in a concomitant charge density wave. These so-called stripe solutions have been confirmed later on by more sophisticated numerical methods. Within a density matrix renormalization group (DMRG) approach White and Scalapino [9, 10, 11] have found stable domain wall solutions in the physically relevant doping regime of the tJ-model. The stripe stability in the tJ-model has also been investigated using exact diagonalization [12, 13] as well as quantum and variational Monte Carlo techniques [14, 15]. Besides the HF approach a variety of methods has also been applied to Hubbard-type models in order to investigate the possibility of charge and spin order. Fleck and collaborators [16] have found stable stripes using dynamical mean-field theory (DMFT) 
and a cluster perturbation approach has been applied in Ref. [20]. From an ab-initio perspective stripe order in lanthanum cuprates has also been shown to be stable in LDA+U calculations [21].

Here we present a short update of the current experimental situation (Sec. 2) followed by an overview over static and dynamical properties of stripes $[22,23,25$, $26,27,72,73,28,29,30$ ] obtained within the timedependent Gutzwiller approximation (TDGA)[133, 134, 135, 136] and phenomenological models. In Sec. 3 we first derive the parameter set for the extended Hubbard model which in the following sections allows us to make quantitative comparison with experimental data. We then show in Sect. 4 that the Gutzwiller approximation (GA) of this model in fact leads to stable stripe ground states which allows for the doping dependent evaluation of the corresponding incommensurate spin response. In Sec. 5] we discuss the electronic structure and various transport properties of stripes. Sec. 6 focusses on the momentum and frequency dependent spin excitations where we also address recent resonant inelastic x-ray scattering experiments. Sec. 7 presents results for the charge excitations from stripe ground states, focussing on the optical conductivity. Finally, we show in Sec. 8 how our Gutzwiller variational theory can be extended towards a dynamical description of stripes before we summarize our discussion in Sec. 9

In the following section we start with an update of the experimental situation.

\section{Brief Experimental Review}

First evidence for stripe order in HTSC's came from elastic neutron scattering experiments by Tranquada and collaborators [31, 32, 33]. They observed a splitting of both spin and charge order peaks in $\mathrm{La}_{1.48} \mathrm{Nd}_{0.4} \mathrm{Sr}_{0.12} \mathrm{CuO}_{4}$ (LNSCO) which resembled similar data in the nickelates where both incommensurate antiferromagnetic (AF) order [34, 36] and the ordering of charges [35, 36] has been detected by neutron scattering and electron diffraction, respectively. In Ref. [36] it was shown that the magnetic ordering displays itself as an occurence of first and third harmonic Bragg peaks whereas the charge ordering is associated with second harmonic peaks. Meanwhile analogous static stripe order has also been detected in $\mathrm{Eu}$-codoped lanthanum cuprates (LECO) [38] and $\mathrm{La}_{2-x} \mathrm{Ba}_{x} \mathrm{CuO}_{4}$ (LBCO) [37, 50] which all display a LTT lattice distortion. That the latter is not necessarily a condition precedent to the formation of stripes has recently been demonstrated by applying pressure to a $\mathrm{La}_{1.875} \mathrm{Ba}_{0.125} \mathrm{CuO}_{4}$ sample. This restores the structure to fourfold lattice symmetry keeping symmetry broken electronic stripe states[39]. Moreover, charge and spin stripe order can also be induced in the LTO phase of impurity $(\mathrm{Cu}, \mathrm{Zn}, \mathrm{Fe}, \mathrm{Ga})$ substituted lanthanum cuprates [40] without the need of applying pressure. Whereas charge order in the neutron and hard $\mathrm{X}$-ray scattering [41] experiments mentioned above has only been detected indirectly via the associated lattice modulation, more recent soft resonant x-ray scattering studies on LBCO [42] and LECO [43] have directly revealed the spatial modulation of charge in these systems.

The incommensurability $\delta$ (defined as the shift of the magnetic Bragg peaks from the antiferromagnetic (AF) wave-vector $Q_{A F}$ in reciprocal lattice units) in samples with long range stripe order follows the socalled 'Yamada-Plot' [44] and depends linearly on doping $\delta=n_{h}$ up to $n_{h} \approx 1 / 8$. Here $n_{h}$ is the number of added holes per planar $\mathrm{Cu}$ with respect to the parent insulating compound. This behavior is compatible with stripe like modulations of charge and spin having a linear concentration of added holes $v=1 / 2$ (so-called half-filled stripes) as we will discuss in more detail in Sec. 4

Evidence for some kind of stripe order in other HTSC materials is based on the doping dependence of the low energy spin response. In non-codoped lanthanum cuprates (LCO) the incommensurability $\delta$ (defined in terms of the shift of the low energy magnetic scattering from $Q_{A F}$ ) also follows the 'Yamada-Plot' of the samples with long-range order. This suggests that the static stripe order is replaced by some kind of 'fluctuating order' in the Nd- (or Ba-, Eu-) free samples. Above $n_{h} \approx 1 / 8$, the incommensurability stays essentially constant but the intensity of the low energy spin fluctuations decreases and vanishes at the same concentration where superconductivity disappears in the overdoped regime [45]. This behavior is mirrored at low doping in that superconductivity, in LSCO materials, disappears upon decreasing doping exactly at the point in which incommensurate scattering parallel to the $\mathrm{CuO}$ bond disappears. 449] This strong correlation between superconductivity and low energy incommensurate scattering parallel to the $\mathrm{CuO}$ bond suggesting an intimate relation between both phenomena and provides a strong motivation to understand stripe physics in cuprates.

Once superconductivity disappears by decreasing doping below $n_{h} \approx 0.055$ in lanthanum cuprates incommensurate scattering does not disappear entirely but rotates by $45^{0}$ [46, 47, 48, 49] to the diagonal direction. Additionally the orthorhombic lattice distortion allows one to conclude that the elastic diagonal mag- 
netic scattering is one-dimensional with the associated modulation along the orthorhombic $b^{*}$-axis, supporting again the picture of stripe formation. When $\delta$ is measured in units of reciprocal tetragonal lattice units in both the vertical and diagonal phase it turns out that the magnitude of the incommensurability numerically coincides across the rotation leading to a linear relation $\delta=n_{h}$. Upon approaching the border of the AF phase at $n_{h}=0.02$ the incommensurability approaches $\epsilon=n_{h}$ [51] where $\epsilon$ is measured in units of reciprocal orthorhombic lattice units, thus $\epsilon=\sqrt{2} \delta$. The crossover from diagonal to vertical incommensurate spin response is so far only observed in lanthanum cuprates whereas in all other HTSC compounds the scattering is always along the $\mathrm{Cu}-\mathrm{O}$ bond direction. Despite this peculiarity of LCO other experiments point to universal behavior.

A linear relationship between doping and incommensurability is also observed in $\mathrm{YBCO}_{6+x}$ although the curve falls below the one of lanthanum cuprates [52, 53, 54]. Apart from details, this suggests that stripe physics is a universal phenomenon in cuprates.

Upon increasing frequency of spin excitations the dispersion develops a spectrum ('hour glass') which is similar in many high- $\mathrm{T}_{c}$ cuprates again suggesting universality. At a certain frequency $E_{s}$ the incommensurate branches merge [55] at the AF wave-vector and for even higher energies the excitations become incommensurate again. The 'hour glass' spectrum has been detected in the vertical [56, 58, 59, 60] and diagonal phase $[62,64]$ of lanthanum cuprates, the YBCO compounds [65, 66, 67, 68, 69] and $\mathrm{Bi}_{2} \mathrm{Sr}_{2} \mathrm{CaCu}_{2} \mathrm{O}_{8+y}$ [70, 71].

Magnetic fluctuations which dispersion follows the 'hour-glass' shape naturally arise from stripe correlations in the ground state $[72,73,74,75,76,77,78,79$, 80, 81, 82, 83, 29] (cf. Sec. 6). Basically a Goldstone mode arises from each of the incommensurate wave-vectors and disperses in a cone-shaped structure to higher energies. However, due to disorder and peculiar magnetic couplings across the stripes (as supported by recent inelastic neutron scattering (INS) experiments on $\mathrm{La}_{2-x} \mathrm{Sr}_{x} \mathrm{CoO}_{4}$ [84]) the weight of the outwards dispersing contribution is strongly suppressed so that the excitations which dominate the spectral weight disperse towards the AF wave-vector $Q_{A F}$. The corresponding excitation energy $E_{S}$ at $Q_{A F}$ is essentially determined by the spin coupling across the antiphase domains. Since at high energies the excitations should again resemble those of the (undoped) AF one finds an outwards dispersing intensity also above $E_{s}$.

While apparently the spectrum of spin excitations is compatible with stripe correlations the problem is the detection of an associated charge order in the non- codoped lanthanum cuprates, YBCO and bimuthates. Experimental data of local probes like NQR [85, 86, 87], NMR [88], EXAFS [182], and X-ray microdiffraction [183] are suggestive of the formation of electronic inhomogeneities, possibly induced by magnetic field [89], but cannot provide evidence for long-range order. In this context it is remarkable that due to refinements in the experimental technique Haase et al. [88] where able to demonstrate a correlation of charge and density variations on short length scales.

One way to reconcile the 'stripe-like' magnetic excitation spectrum with the absence of long-range charge order is the notion of a charge nematic [90] which (in the charge channel) breaks rotational but not translational invariance. This concept is extremely useful in detwinned YBCO where both neutron scattering [68, 69] and thermoelectric transport [91] data show pronounced in-plane anisotropies but may also apply to the spin-glass phase of lanthanum cuprates where, as mentioned above, the incommensurate spin scattering is one-dimensional.

Due to the absence of clear signatures of charge ordering in other than co-doped lanthanum cuprates alternative theories have been proposed in order to account for the incommensurate spin scattering and the associated 'hour glass' magnetic spectrum [92, 93, 94, 95, 96, 97, 98, 99, 100]. Nonetheless there are a number of fingerprints in spectroscopic probes which support the presence of incommensurate charge scattering. For example, the anomalous lineshape and temperature dependence of the bond-stretching phonons in various cuprate materials (cf. Ref. [101] and references therein) can be attributed to the influence of stripes. For example, it has been proposed that the softening of these phonons at certain momenta is due to the influence of charge stripe fluctuations on the phonon self-energy [102] In addition one may also have a Kohn-type anomaly due to the nesting along the half-filled stripe [103]. Furtheron, the scattering of charge carriers by incommensurate charge fluctuations should induce a reconstruction of the Fermi surface [20, 104, 105]. Angle-resolved photoemission experiments (ARPES) on LSCO [106, 107] have indeed shown the appearance of straight Fermi surface (FS) segments around the M-points of the Brillouin zone as expected for a striped ground state. However, one peculiar feature of these experiments concerns the fact that the stripe-like FS was obtained from the momentum distribution $n_{\mathbf{k}}$, by integrating the spectral function over a broad energy window $(\sim 300 \mathrm{meV})$. On the other hand, upon following the momentum dependence of the lowenergy part of the energy distribution curves a large FS was found corresponding to the LDA band-structure and 
fulfilling Luttinger's theorem. We will address this dichotomy of stripe-like spectral features at large energies and a 'protected' FS at low energies in Sec. 8 .

In LECO, where static charge order has been detected with RIXS [43], the associated reconstruction of the FS has indeed been resolved by ARPES. Moreover, a recent series of magnetotransport experiments [110, 111, 112, 113] have revealed quantum oscillations suggestive of the formation of electron pocktes due to a FS reconstruction. It has been shown by Millis and Norman [114] that a charge density wave ground state with intermediate values of the stripe order parameter is in qualitative agreement with the quantum oscillation data. A recent comparative study of thermoelectric properties in YBCO and LECO [115] also provides strong evidence that the Fermi surface reconstruction in YBCO is associated with stripe correlations. In the context of transport experiments one should also mention the Nernst effect which in hole doped HTSC's above T $_{c}$ usually yields a large positive signal [116] and which has been considered as indicative of fluctuating superconductivity. However, in addition also a FS reconstruction contributes to the Nernst signal and both contributions have been resolved in LNCO and LECO [117. These authors have attributed the high temperature signal to originate from changes in the FS, an interpretation which has recently been challenged in Ref. [118]. A theoretical analysis of the Nernst effect in terms of stripe order can be found in Refs. [119, 120].

Scanning tunneling microscopy (STM) provides additional evidence (limited obviously to the surface) for charge order in cuprate superconductors. Corresponding measurements performed on bismuthate and oxychloride superconductors have revealed a complex modulation of the local density of states (LDOS) both in the superconducting (SC) state [121, 122, 123, 124, 125, 126] and above $T_{c}[126,127,[128]$. In both cases one observes peaks in the Fourier transform of the real space LDOS at wave-vectors $Q=2 \pi /\left(4 a_{0}\right) \ldots 2 \pi /\left(5 a_{0}\right)$ suggestive of checkerboard or stripe charge order. However, it is important to distinguish between the case where these peaks are non-dispersive in energy (and thus signature of 'real' charge order) or the situation where they follow a bias-dependent dispersion due to quasiparticle (QP) interference. In the latter case the spatial LDOS variations can be understood from the so-called octet model [121, 129] which attributes the modulations to the elastic scattering between the high density regions of the Bogoljubov 'bananas' in the superconducting state. Recent STM investigations [130,131] may resolve this apparent conflict since they suggest that both, dispersive and non-dispersive scattering originates from different regions in momentum and energy space. The states in the nodal region which are well defined in k-space and undergo a transition to a $d$-wave SC state below $T_{c}$ are then responsible for the low energy QP interference structure of the LDOS, whereas the ill-defined kspace 'quasiparticle' states in the antinodal regions are responsible for the non-dispersive $\mathrm{CO}$ above some energy scale $\omega_{0}$. As mentioned before we will address the issue of high energy electronic order in Sec. 8 .

\section{Model and parameters}

Moment formation in a spin-density wave solution involves the large Hubbard $U$ scale and modifies the electronic structure of the system over a large energy range. Therefore, it is not a priori obvious that the reduction of a multiband model to a more simplified model hamiltonian will retain the relevant features in the spectra, especially when one is concerned with high frequency excitations. A suitable method in order to deal with this problem has to fulfill essentially the following two conflicting criteria: $(a)$ it should be sufficiently accurate in order to account for the Hubbard-type correlations of the model and $(b)$ it should allow for the investigations of large systems in order to provide a sufficient resolution for the correlation functions in momentum space.

Subsequent to the first stripe calculations [5, 6, 7, 8], investigations of collective excitations have been performed within the Hartree-Fock (HF) approximation of the one-band Hubbard model supplemented with RPA fluctuations [137, 138, 139, 140, 141]. However, in case of the HF approximation[142] it turns out that the stripe solutions relevant for cuprates are only favored for unrealistic small values of $U / t \approx 3 . .5$ whereas a ratio of $U / t \approx 8$ is required to reproduce the magnon spectrum of LCO (cf. below).

In order to enable a quantitative comparison with experiment, the results we will discuss in the following sections will be mostly based on the Gutzwiller approximation (GA) [132] supplemented with Gaussian fluctuations [133, 134, 135, 136] (so-called time-dependent GA [TDGA]).

We consider the one-band Hubbard model

$$
H=\sum_{i, j, \sigma} t_{i j} c_{i, \sigma}^{\dagger} c_{j, \sigma}+U \sum_{i} n_{i, \uparrow} n_{i, \downarrow},
$$

where $c_{i, \sigma}\left(c_{i, \sigma}^{\dagger}\right)$ destroys (creates) an electron with spin $\sigma$ at site $i$, and $n_{i, \sigma}=c_{i, \sigma}^{\dagger} c_{i, \sigma} . \quad U$ is the on-site Hubbard repulsion and $t_{i j}$ denotes the hopping parameter between sites $i$ and $j$. We restrict to hopping between 
nearest $(\sim t)$ and next-nearest $\left(\sim t^{\prime}\right)$ neighbors. For high$\mathrm{T}_{c}$ cuprates the value of $U$ can be estimated from the magnon dispersion of the undoped system [144, 145]. In fact, within spin-wave theory (SWT) applied to the Heisenberg model (corresponding to $U / t \rightarrow \infty$ ) the magnon excitations are given by

$$
\hbar \omega_{q} \propto \sqrt{1-\left[\cos q_{x}+\cos q_{y}\right]^{2} / 4}
$$

and thus the dispersion vanishes along the magnetic Brillouin zone. Including quantum fluctuations beyond SWT [143] leads to excitations with lower energy at $(\pi, 0)$ than at $(\pi / 2, \pi / 2)$, contrary to what is observed in undoped cuprate superconductors [144, 145]. It has been argued that in cuprates corrections to the Heisenberg model arising as higher orders in a $t / U$ expansion are relevant [146, 147, 148, 149, 144]. The most important of such corrections is a term which cyclically exchanges four spins on a plaquette. A sizable value for this term has been revealed by analyzing phononassisted multimagnon infrared absorption [149] and the dispersion relation measured with INS [144, 145] shown in Fig. 1. In particular, the dispersion in the $(\pi, 0)$ and $(\pi / 2, \pi / 2)$ is mainly due to this term. In case of the Hubbard model the magnetic zone edge dispersion is due to the finiteness of $U / t$. For moderate values of $U / t$ this leads to dispersion which decreases from $(\pi, 0)$ to $(\pi / 2, \pi / 2)$ along the magnetic zone boundary whereas for large $U / t$ the behavior of the spin-1/2 Heisenberg model is recovered[150].

Fig. 11 1 shows the $U / t$ dependence of the magnetic excitations for the half-filled AF as compared to INS data from Ref. [144]. The dispersion along the magnetic zone boundary can be accurately fitted by a value of $U / t=8$ where the absolute energy scale is fixed by the nearest neighbor hopping $t=360 \mathrm{meV}$. Panel b of Fig. 1 displays the dependence of the spin excitations on the next-nearest neighbor hopping $t^{\prime} / t$. For small to moderate values of $t^{\prime} / t$ the zone boundary dispersion is only slightly dependent on this parameter, however, significant softening occurs for $t^{\prime} / t=-0.5$. The increasing frustration between nearest and next-nearest neighbor magnetic interactions drives the system towards an $1 \mathrm{D}-\mathrm{AF}$ instability the precursor of which is seen as a magnetic mode softening at $\mathbf{q}=(\pi, 0)$.

Further information on the parameter $t^{\prime} / t$ can be obtained from the optical conductivity which in the inset to Fig. 1b is shown for the same parameters as used in the main panel. The lowest energy excitation $\Omega_{\text {min }}$ for the half-filled system is shifted up with increasing $\left|t^{\prime} / t\right|$ and the experimental value of $\Omega_{\text {min }} \approx 2.1 \mathrm{eV}$ for $\mathrm{LCO}$ [151, 152] is accurately reproduced for the parameter
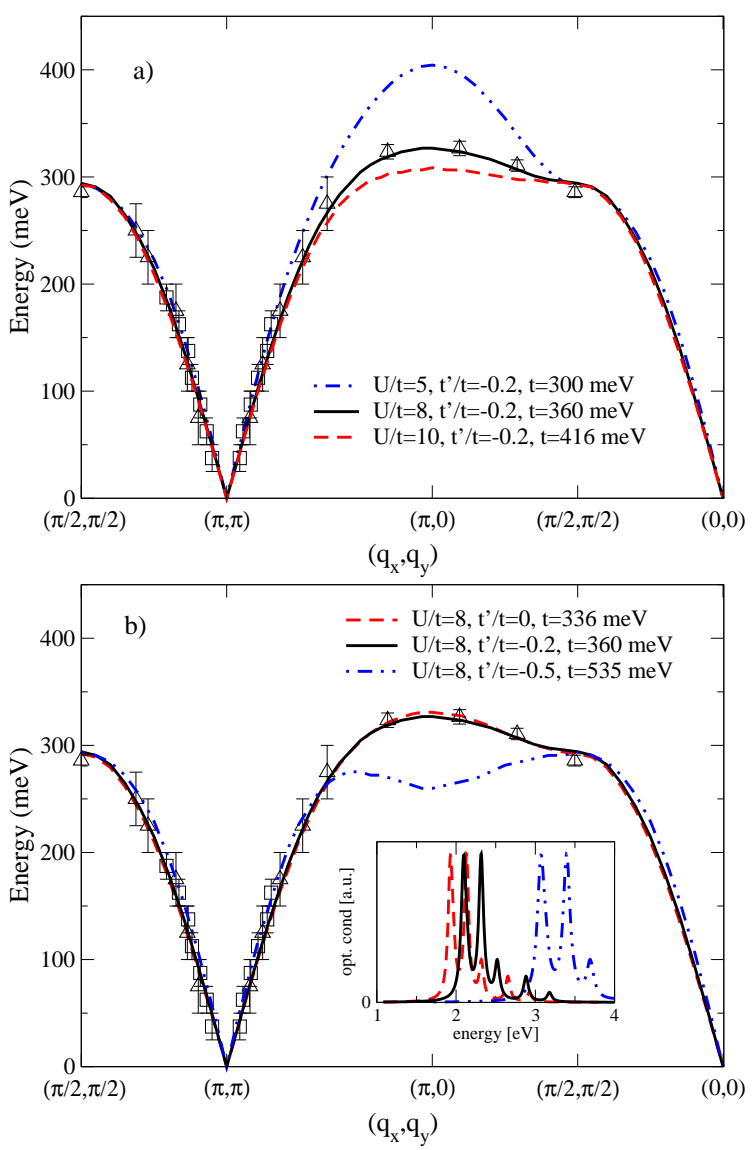

Figure 1: Energy and wave vector dependence of magnetic excitations in the half-filled system as obtained from the TDGA for (a) $t^{\prime} / t=$ -0.2 and varying $U / t$; (b) $U / t=8$ and varying $t^{\prime} / t$. Squares and triangles correspond to data points from INS experiments on $\mathrm{LaCuO}_{4}$ by Coldea et al. [144]. The inset to (b) shows the optical conductivity for the same parameters as used in the main panel (b).

set $U / t=8, t^{\prime} / t=-0.2, t=360 \mathrm{meV}$. In the following section we will show that a value of $t^{\prime} / t=-0.2$ is also appropriate to account for the doping dependent incommensurability of lanthanum cuprates [44] based on a striped ground state.

Note that some few results discussed in the present review (Secs. 4.1,7) are also obtained within the threeband model which for copper $d_{x^{2}-y^{2}}$ and oxygen $p_{x, y}$ orbitals includes the associated hopping processes, orbital energies, and intra- and interorbital Coulomb repulsions. We have taken these parameters either from first principle computations [167, 168] or from more empirical considerations [156]. 


\section{Stripe stability and static properties}

\subsection{Charge and spin structure}

Stripes are characterized by one-dimensional antiphase boundaries for the AF order which also host the doped holes. These textures can be understood as the strong coupling remnant of an instability in the spin channel. It can be shown [154] that for dispersion relations relevant for cuprates the orientation is either along the copper-oxygen (vertical) or the diagonal direction. The domain walls can either reside on lattice sites or bonds and these two prototypical stripe solutions for both vertical and diagonal orientations are sketched in Fig. 2]

By symmetry, for site-centered (SC) stripes the magnetization vanishes on the domain wall whereas for bond-centered (BC) stripes these are build up from ferromagnetically aligned nearest-neighbor spins. For diagonal BC stripes this leads to the situation that configurations with $d=$ even acquire a macroscopic magnetization in contrast to vertical BC stripes where the ferromagnetic bonds alternate along the domain wall. Diagonal BC stripes can be viewed as a realization of the staircase structures proposed by Granath [155] with step length $l=2$.

Fig. 3] shows the charge and spin density for $d=$ $4 \mathrm{BC}$ vertical stripes (doping $n_{h}=1 / 8$ ) obtained within the 3-band model and parameters taken from Ref. [156]. A similar charge distribution was predicted in Ref. [25] and found to be in excellent agreement with a charge sensitive probe by Abbamonte and collaborators 42]. However, these authors could not determine if the stripes where $\mathrm{Cu}$ centered or $\mathrm{O}$ centered. More recently Davis and collaborators 157] have imaged glassy stripes which indeed are centered on $\mathrm{O}$ as predicted[25]. Taken together these experiments give us amplitude and phase information of stripes in excellent agreement with Ref. [25] thus showing that it is possible to obtain and even predict realistic information on the intermediate scale physics of cuprates.

The Fourier transform of the charge $\left(\propto \rho_{Q}\right)$ and spin distribution $\left(\propto m_{Q}\right)$ determines Bragg peak weights $\left(\propto \rho_{Q}^{2}, m_{Q}^{2}\right)$ in scattering experiments (for the definitions see Ref. [158]) as shown in Fig. 4 Disregarding the difference in cross section for different processes (magnetic neutron scattering vs. X-ray or nuclear neutron scattering) we see that Bragg weights for the charge are practically 3 orders of magnitude smaller than those for the $\mathrm{Cu}$ spins. This is due to very soft charge distribution shown in Fig. 3 with respect to the spin distribution and explains why it has been so hard to detect charge ordering. a)

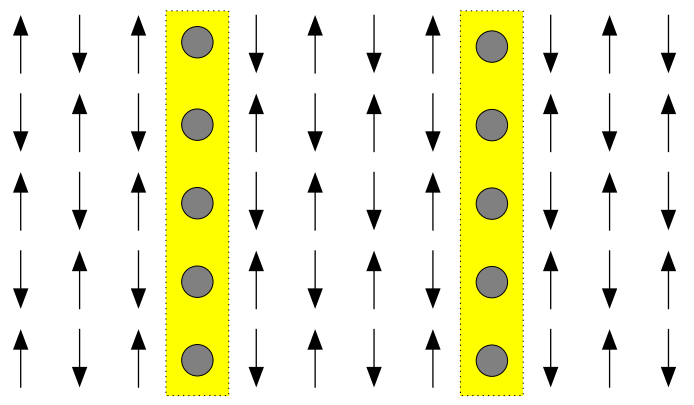

b)

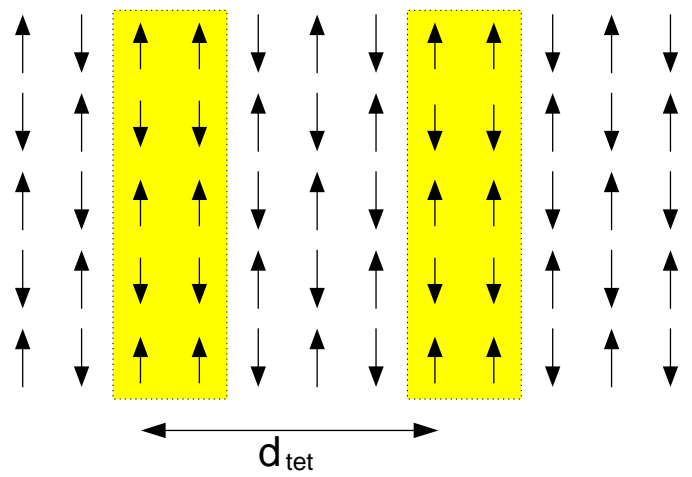

c)

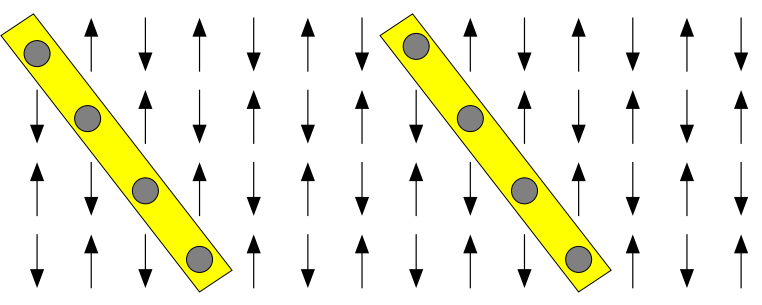

d)

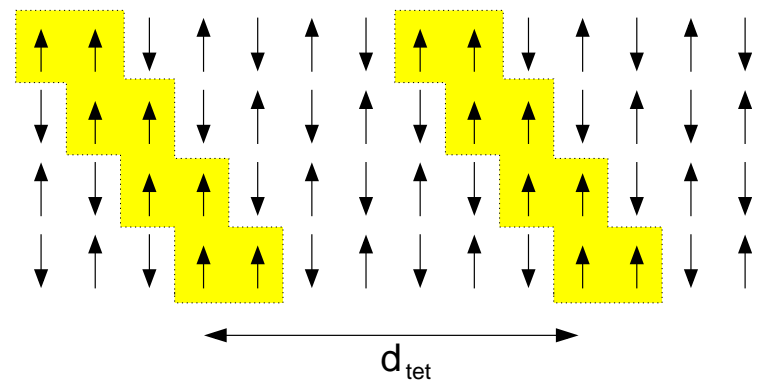

Figure 2: Spin structure of vertical $(a, b)$ and diagonal stripe textures. The doped holes mainly reside on the (shaded) domain walls. In both cases the prototypical structures comprise site-centered (a,c) and bond-centered structures (b,d). The distance between charge stripes, measured along the copper-oxygen bond direction is denoted as $d$ in units of the tetragonal lattice constant $a_{\text {tet }}$. 


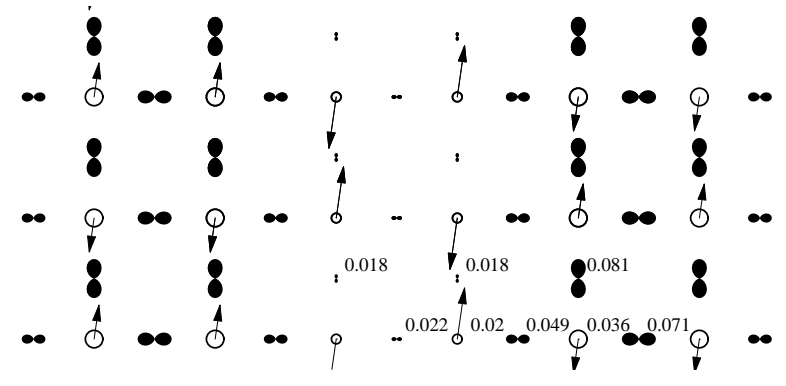

Figure 3: Charge and spin density for $d=4 \mathrm{BC}$ vertical stripes. Doping $n_{h}=1 / 8$. Open circles ( $p$ orbitals) represent $\mathrm{Cu}(\mathrm{O})$ sites. The numbers and the size of symbols represent the excess charge whereas the spin density is proportional to the length of arrows.
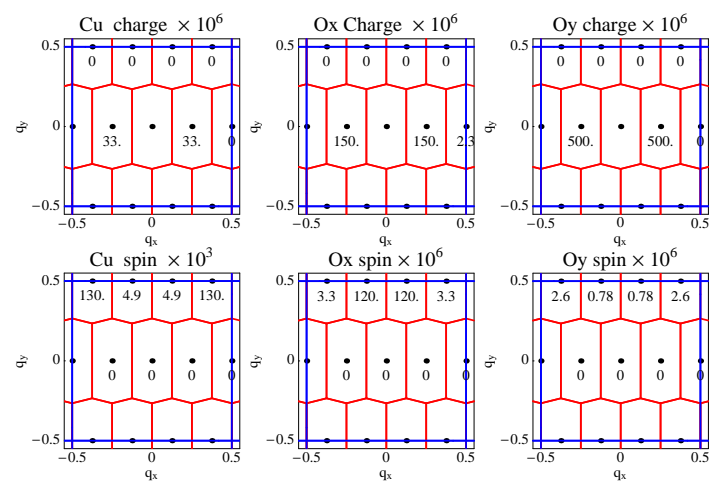

Figure 4: Bragg weights $m_{\boldsymbol{O}}^{2}, \rho_{\boldsymbol{O}}^{2}$ at reciprocal lattice vectors (indicated by the dots) for $n_{h}=1 / 8$ and $d=4$ stripes. The polygons around each point are the reduced magnetic Brillouin zones. We use reciprocal lattice units of the fundamental Brillouin zone. $\mathrm{O}_{x}\left(\mathrm{O}_{y}\right)$ are the oxygens in the bonds perpendicular (parallel) to the stripe.

\subsection{Stripe stability and incommensurability}

The task is now for a given parameter set and doping to variationally determine the lowest energy state among the various stripe states and to compare with other possible solutions. This has been accomplished in Refs. [27, 28, 30] for the one-band hamiltonian Eq. 1 and in Ref. [25] also for the three-band model. Fig. 5 displays the binding energy per hole

$$
e_{h}=\frac{E_{A F}\left(N_{h}=0\right)-E_{t e x t}\left(N_{h}\right)}{N_{h}}
$$

for the energetically most competing structures, namely stripes, spirals and spin polarons. Here $E_{A F}\left(N_{h}=0\right)$ denotes the energy of the undoped $\mathrm{AF}$ and $E_{\text {text }}\left(N_{h}\right)$ is the energy of a given texture obtained for doping the system with $N_{h}$ holes. We can also define the filling factor $v$ of stripes which corresponds to the concentration of holes per unit cell along the stripe. This can be related to the hole concentration $n_{h}$ and the distance (in $a_{t e t}$ lattice units) between charge stripes $d$ as $v=n_{h} \cdot d$. From the inset to Fig. 5a it can be seen that for $U / t=8$ and $t^{\prime} / t=-0.2$ low doping vertical stripes have $v_{\text {opt }} \approx 0.55$ whereas the minimum of diagonal SC (BC) domain walls is at $v_{\text {opt }}=1(0.75)$. We will see below that $v_{\text {opt }}$ determines the slope of the Yamada plot at low doping.

We find that for the one-band model, vertical BC and SC stripes are also practically degenerate in energy in agreement with Refs. [9, 16, 163]. For definiteness we mainly restrict ourself to the $\mathrm{BC}$ case because these textures constitute the more stable configuration at $n_{h}=1 / 8$ in the more accurate three-band model [25] and in first principle computations[21]. However, one should keep in mind that all energetic considerations below hold equally for SC vertical stripes.

From the inset to Fig. 5h it turns out that diagonal $\mathrm{BC}$ stripes are practically (accidentally) degenerate in energy with vertical stripes. The energy of the DSC texture is $\approx 0.02 t$ per hole above. These small energy differences should not be significant given the simplicity of the model. A precise determination of the relative stability of the different phases would require at least multiorbital effects, inclusion of both long-range Coulomb interactions and coupling of the holes to the tilts of the $\mathrm{CuO}_{4}$ octahedra. The latter have been shown to play a major role in the stabilization of vertical $v s$. diagonal stripes[159].

In Fig. 5 the dot-dashed line corresponds to the envelope of the stripe binding energy curves for different periodicites $d$. The solid (dashed) lines show $e_{h}$ for diagonal (vertical) spiral solutions which apparently are unstable towards phase separation at low doping. The horizontal line is the energy of the phase separated state obtained from the Maxwell construction. The respective stability of stripes and uniform spirals is determined by the ratio between next-nearest neighbor and nearest neighbor hopping $t^{\prime} / t[30,161,162]$. For $t^{\prime} / t=-0.2$ stripes are stable over the whole doping range up to $n_{h} \approx 0.28$ where their energy becomes degenerate with that of vertical spirals. In contrast, it is found that with increasing $\left|t^{\prime} / t\right|$ stripes with significant spin canting start to dominate the phase diagram and for even larger $\left|t^{\prime} / t\right|$ also checkerboard textures may become the ground state [28, 30]. Also the optimum filling $v_{o p t}$ changes with $t^{\prime} / t$ as expected according to the results of Ref. [27]. The influence of a next-nearest neighbor hopping term on the stripe stability has been previously investigated by various methods. From the DMRG approach applied to the $\mathrm{t}-\mathrm{t}^{\prime}-\mathrm{J}$ model [11] it turned out that a negative $t^{\prime} / t$ can suppress both the formation of stripes and pairing cor- 


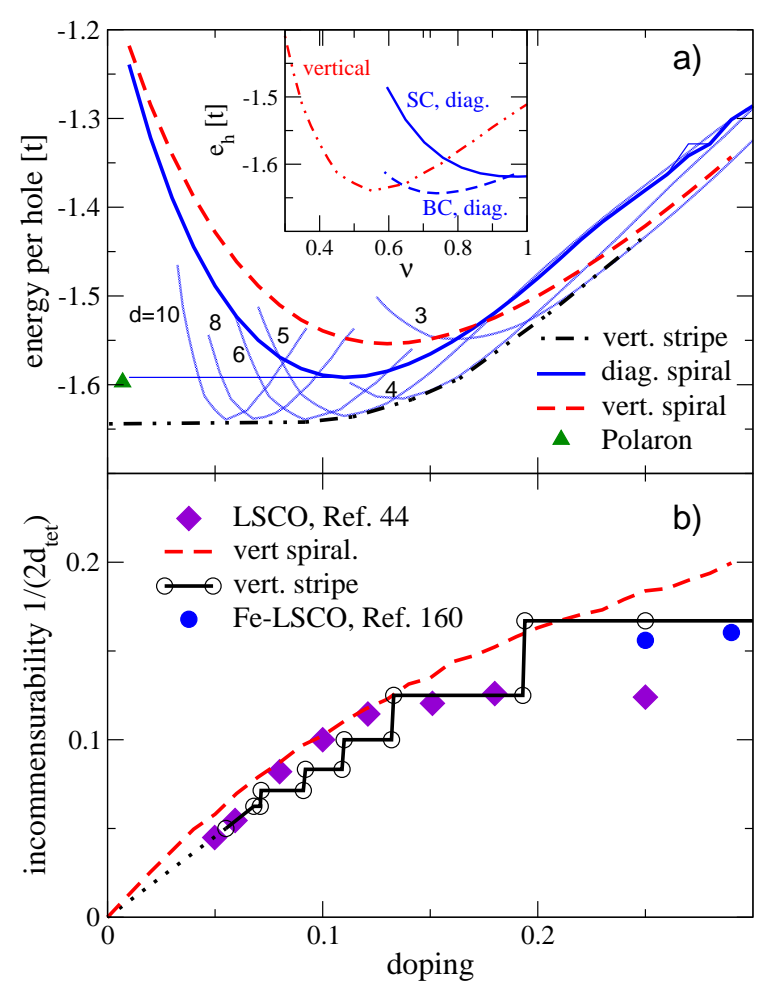

Figure 5: (a) Binding energy per hole $e_{h}$ as a function of doping for stripes, uniform spirals and spin polaron. The inset shows $e_{h}$ for $d=$ 10 vertical (dashed-dotted), bond centered diagonal (dashed), and sitecentered diagonal (solid) stripes as a function of the filling factor $v$. (b) Incommensurability $1 /(2 d)$ of vertical stripes and uniform spirals as a function of doping. Diamonds are data for LSCO from Ref. [44]; full dots are data for Fe-codoped LSCO from Ref. [160]. Results are obtained from the one-band model with parameters: $U / t=8$ and $t^{\prime} / t=-0.2$.

relations. A weakening of stripe tendencies for $t^{\prime} / t<0$ in the same model was also found with exact diagonalization [13] and in the Hubbard model with DMFT [16], and the HF approximation. [17, 18, 19] All these calculations suggest that static stripes are destabilized when the ratio $t^{\prime} / t$ becomes negative. This may indicate a more dynamical character of stripes in some systems like $\mathrm{Tl}$ and $\mathrm{Hg}$ based compounds.

With regard to the experimental evidence of stripe correlations in lanthanum cuprates it turns out that a ratio of $t^{\prime} / t=-0.2$ can account for the doping dependent incommensurability $\delta$ observed in these compounds [44]. For vertical stripes this quantity is related to the modulation of the AF order and thus given by

$$
\delta=1 /(2 d)=n_{h} /(2 v)
$$

in units of $2 \pi / a_{t e t}$ where $a_{t e t}$ is the tetragonal lattice constant. The charged core of the stripe has a characteristic width $\xi$ so that when the charge periodicity $d$ is larger than $\xi$ there are negligible interstripe interactions and doping proceeds by increasing the number of stripes. In this regime the number of stripes for a fixed number of holes is optimized when $v=v_{\text {opt }}$. Thus $v_{\text {opt }}$ determines the slope of the incommensurability as a function of doping, according to Eq. (4) and $v_{\text {opt }}=0.55$ implies $\delta=0.91 n_{h}$ in good agreement with the Yamada plot. The regime $d>\xi$ is characterized by the fact that $e_{h}$ at the minimum is independent of doping or $d$. From Fig.5 we find $\xi=4$ lattice units which coincides with a direct examination of the charge profile. For $d \lesssim \xi$ stripes completely cover the plane and increasing further the number of stripes becomes energetically costly. Therefore doping proceeds by increasing the charge of stripes and $\delta$ becomes constant. The crossover in doping between the two regimes occurs when $n_{h}=v_{\text {opt }} / d \sim 1 / 8$ in good agreement with the Yamada plot in LCO.

As can be seen from Fig. 5b the incommensurability for stripes shows a staircase structure where the steps occur at the crossing of the corresponding energy curves (cf. Fig. 5a). For small doping $(d>\xi)$, since interstripe interactions are negligible, one can produce a practically continuous curve by considering combinations of solutions with periodicity $d$ and $d+1$. As explained above this is not any more convenient for $d \lesssim \xi$ and the additional holes are doped into existing stripes producing the plateau in the incommensurability.

Contrary to the stripes, one observes a continuous increase of $\delta\left(n_{h}\right)$ in case of uniform spirals . Nevertheless, both phases show similar evolution of incommensurability with doping and indeed fairly similar values of $\delta\left(n_{h}\right)$ up to $n_{h} \sim 1 / 8$. For larger doping this agreement is lost due to the tendency of stripes to make wide plateaus.

We have explained the Yamada plot in terms of well formed stripe textures. It is remarkable that the incommensurabilities, both for stripes and spirals, are not far from what one would obtain from an analysis of the momentum dependent susceptibility of the Fermi liquid phase [153]. In fact, if one adiabatically decreases the interaction both uniform spirals and stripes originate from the same magnetic instability [154]. One can call this a "weak coupling" instability. On the other hand within the time-dependent Gutzwiller approximation, the Hubbard interaction $U$ is strongly screened by vertex corrections. Therefore a quite large value of $U$ is needed in order to make the Fermi liquid unstable and the instability is better characterized as "intermediate coupling".

The time dependent Gutzwiller analysis shows that it is the plateau in the spin susceptibility close to $Q_{A F}=$ $(\pi, \pi)$ (cf. Fig. 1 in Ref. [154]) which drives the system 
unstable towards spiral or stripe order for sufficiently large on-site repulsion $U / t$.

Figure $5 \mathrm{~b}$ also shows data from more recent neutron scattering experiments [160] on Fe-LSCO. In these overdoped samples an elastic incommensurate spin response was found close to the dominant nesting vectors as extracted from ARPES experiments. It was thus concluded that the induced incommensurate response signals an inherent instability of the itinerant charge carriers, being different from the low doping stripes arising from localized $\mathrm{Cu}$ spins. However, from Fig. $5 \mathrm{~b}$ it turns out that the data are rather close to the incommensurability curve of stripes obtained for the LSCO parameter set. Remarkably this behavior was predicted in Ref. [25] much before the measurement became available. Since these stripe calculations are based on an itinerant approach, there is no 'dichotomy' between low doping 'localized' spin stripes and large doping itinerant ones, but both appear as different limits of the same model.

Finally we briefly address the incommensurability of diagonal stripes. In tetragonal units the incommensurability is a factor of $\sqrt{2}$ larger than that for vertical stripes given in Eq. (4). The more stable BC diagonal textures have $v_{\text {opt }} \approx 0.75$ so that $\delta_{\text {diag }}=n_{h} /\left(\sqrt{2} v_{\text {opt }}\right) \approx n_{h} / 1.06$. Therefore the doping dependence of $\delta$ for BC diagonal and vertical stripes is almost identical in agreement with neutron scattering data [46, 47, 48, 49]. One should keep in mind the effect of disorder which in the diagonal phase reduces the correlation to the same order or even smaller than the periodicity [47] whereas for vertical stripes the correlation length can reach $150 a_{\text {tet }}$ or around 20 times the magnetic stripe periodicity. This becomes especially important in context of spin excitations from stripes in the spin glass phase (cf. Sec. 6).

\section{Electronic structure and transport properties}

Fig. 6] shows the bandstructure for half-filled $d=4$ bond-centered stripes which are oriented along the ydirection. The electronic states of the undoped AF ordered regions (magnetization per spin $m$ ) give rise to lower and upper Hubbard bands (LHB/UHB) which are separated by $\Delta \sim U \cdot m$. Stripes induce the formation of additional bands in the gap and for partially filled stripes (i.e. $0<v<1$ ) one of them ('active band') crosses the Fermi energy. For the present case of 'half-filled' stripes the crossing occurs at $k_{y}^{F}= \pm \pi / 4$ for $k_{x}=0\left(a_{t e t}=1\right)$. Perpendicular to the domain walls the bands are rather flat with the residual small dispersion due to the overlap between adjacent stripes.

The 'active band' implies a quasi one-dimensional Fermi surface at momentum $k_{y}=\pi / 4$ (cf. Fig. 7b).

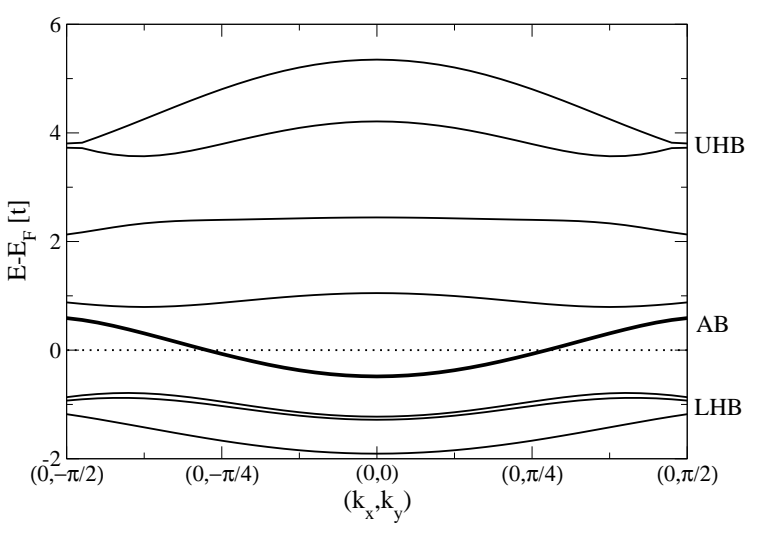

Figure 6: Band structure for $d=4$ bond-centered stripes oriented along the $y$-direction. The dispersion is shown for a cut along the stripe and $k_{x}=0$. The electronically relevant active band (AB) crosses the Fermi energy (doping $n_{h}=0.125$ ) and is in the gap between lower (LHB) and upper (UHB) Hubbard band. The AF modulation in the direction of the stripes leads to a doubling of the periodicity so that $-\pi / 2 \leq k_{y}<\pi / 2$.

The corresponding weight in the full Brillouin zone is concentrated around the $(\pi, 0)$ point which results in the two parallel FS segments. There is also a crossing at $k_{y}=3 \pi / 4$ with less spectral weight and the associated segment is shifted more towards $k_{x}=0$. In LSCO one expects a superposition from $\mathrm{x}$ - and $\mathrm{y}-$ oriented stripes which results in the FS shown in Fig. 7b. Such a prediction is in good agreement with ARPES data [106, 107] and even fine details as the small curvature of the segments due to interstripe hopping are reproduced. It should be mentioned that the experimental data [106, 107] strongly depend on the energy window which is chosen to extract the FS. We will address this point in Sec. 7

As discussed above, stripes are approximately halffilled for doping $n_{h} \lesssim 1 / 8$ and for $n_{h} \gtrsim 1 / 8$ additional holes are doped into the active band. Therefore the chemical potential is expected to be approximately constant for $n_{h} \lesssim 1 / 8$ and decreases for $n_{h} \gtrsim 1 / 8$ in qualitative agreement with the observed behavior[108, 109]. The rate of change of $\mu$ with doping, being a high derivative of the energy, is very sensitive to finite size effects and, moreover, few experimental points are available in this doping range in order to allow for a precise comparison. A rough estimate indicates that the theoretical rate of change of $\mu$ with doping for $n_{h}>1 / 8$ is approximately a factor of 2 larger than the experimental one [108, 109]. This may be attributed to an underestimation of the mass renormalization in mean-field. Another possibility which goes in the right direction is phase separation among the $d=4$ stripe solution and 


\section{a)}
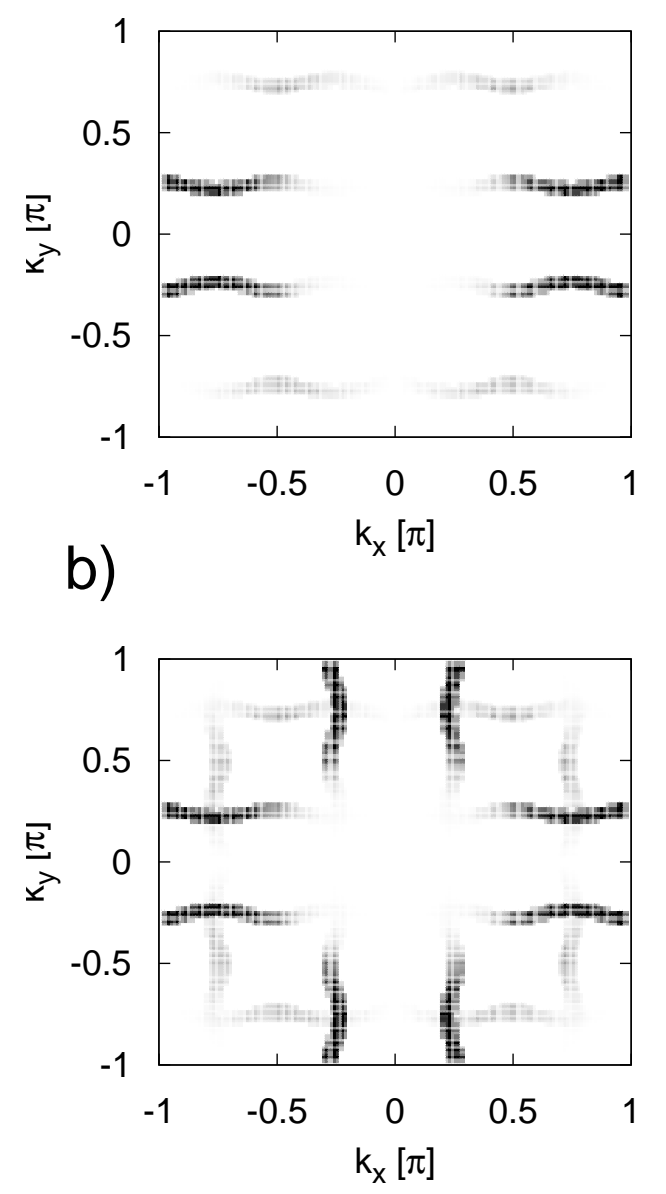

Figure 7: Fermi surface for $d=4$ bond centered stripes at $n_{h}=0.125$. (a) y-axis oriented stripes; (b) superposition of $x$ - and $y$-axis oriented stripes.

the paramagnetic overdoped Fermi liquid which is also suggested by the doping evolution of magnetic excitations (cf. Sec. 6).

We finally note that also anomalies in the Hall and Nernst coefficient can be attributed to the FS reconstruction due to striped ground states [25, 119, 120].

\section{Spin excitations}

Fig. 8 reports constant frequency scans of the imaginary part of the transverse magnetic susceptibility $\chi_{q}^{\prime \prime}(\omega)=-\operatorname{Im} \frac{1}{N} \int e^{i \omega t}\left\langle\mathcal{T} S_{q}^{+}(t) S_{-q}^{+}(0)\right\rangle$ for y-axis oriented $d=4 \mathrm{BC}$ stripes in the magnetic Brillouin zone (cf. Fig. 9).
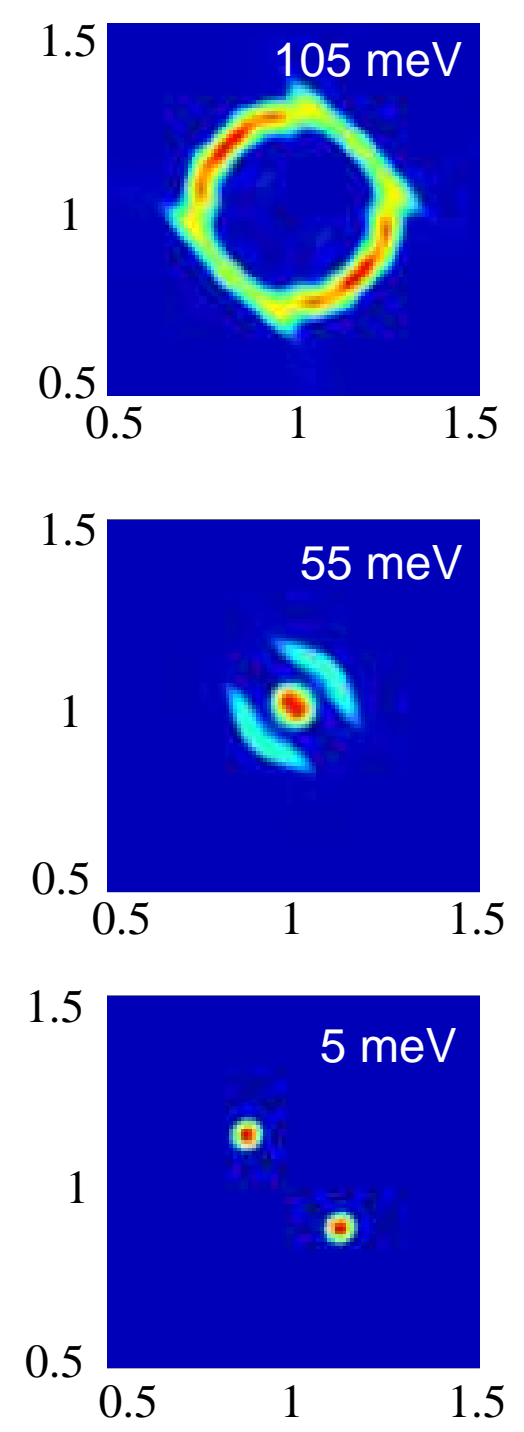

Figure 8: Constant frequency cuts of the magnetic excitation spectrum at $\omega=5,55$, and $105 \mathrm{meV}$ for the $d=4, n_{h}=0.125 \mathrm{BC}$ stripe structure. The momentum space corresponds to the magnetic Brillouin zone which is rotated by $45^{0}$ with respect to the stripe direction (cf. Fig. 9. 


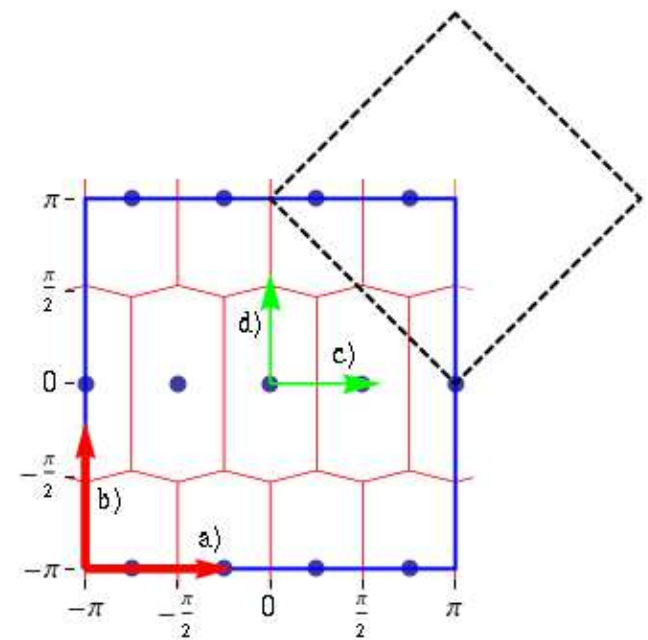

Figure 9: Subdivision of the full Brillouin zone into reduced zones defined from the elementary cell of y-axis oriented $d=4 \mathrm{BC}$ stripes. Arrows (a) ... (d) indicate the scan direction for the magnetic dispersions shown in Figs. 10 and 12 The intensity plots of Fig. 8]are shown within the magnetic Brillouin zone indicated by the dashed square.

The low frequency intensity is concentrated around the wave vectors $Q_{s}=(\pi(1 \pm 1 / 4, \pi)$ corresponding to the spin periodicity of the underlying $d=4$ stripe structure. In fact, it turns out that the weight of the elastic magnetic peaks is much larger at $Q_{s}$ than at the higher harmonics (cf. Fig. 4 where the corresponding weights obtained from the 3-band model calculations are shown). Note also that the magnetic weight vanishes identically on the 'nuclear' Bragg points $Q_{n}=n(\pi / 2,0)$ which only contribute in the charge channel. As a result the dispersion along the cut $\left(q_{x}, \pi\right)$ (cf. Fig. 10, $n_{h}=$ 0.125 ) shows the expected behavior for spin-waves in a striped system [73, 81, 82, 83]. Starting from the Goldstone mode at $Q_{s}$ one observes two branches of spin waves where the large intensity one disperses towards $Q_{A F}=(\pi, \pi)$. The other branch rapidly looses intensity which is analogous to what is found within linear spinwave theory of the Heisenberg model [73, 81, 82, 83] for small ratios of $J_{\perp} / J_{\|}$. Here $J_{\perp}$ denotes the (ferromagnetic) exchange coupling across a stripe whereas $J_{\|}$ denotes the coupling within the AF ordered legs. In addition the intensity of the outwards dispersing branch is strongly reduced by disorder [84].

At $E \approx 55 \mathrm{meV}$ the spin excitations have finally shifted towards the AF wave-vector producing an intense feature at $Q_{A F}$ (middle panel of Fig. 8) which is associated with a saddle-point in the magnetic dispersion [Fig. 10 $\left.\left(n_{h}=0.125\right)\right]$. At the same time one observes the appearance of intensity in the stripe direction starting from $Q_{A F}$. From Fig. $10\left(n_{h}=0.125\right)$ it turns out that this feature is due to a slight softening of the magnetic excitations upon dispersing away from the saddle-point in the direction of the stripe. This softening is absent at lower doping [cf. Fig. $10\left(n_{h}=0.0625\right.$, $\left.\left.n_{h}=0.083\right)\right]$ and indicates the susceptibility towards a magnetic instability along the stripe at higher doping. It has been argued [73] that this roton like minima explains INS in untwinned samples of YBCO by Hinkov and co-workers [61].

Finally, at higher energies the excitations again spread out and form a ring-shaped feature around $Q_{A F}$ with a small anisotropic intensity modulation (Fig. 8 . upper panel). Note that our spectra are for onedimensional stripes without any orientational average so that $\mathrm{C}_{4}$ symmetry is broken. The resulting quasi twodimensional excitation spectra above the saddle-point energy are in marked contrast to the one-dimensional characteristics obtained from localized spin models [74, 75]. This has profound implications on the interpretation of INS data from detwinned samples [68, 69] which also show pronounced 1-D characteristics at low but a 2-D magnetic distribution of magnetic energies at high energies in accord with our result.

The high energy distribution of magnetic intensity can be understood from the energy weighted sum rule

$$
M_{\mathbf{q}}^{1} \equiv \int_{0}^{\infty} d \omega \omega \chi_{\mathbf{q}}^{\prime \prime}(\omega)=-\frac{\pi}{2 N} \sum_{\mathbf{k}, \sigma}\left(\varepsilon_{\mathbf{k}}-\varepsilon_{\mathbf{k}+\mathbf{q}}\right) n_{\mathbf{k}, \sigma}(5)
$$

where $\varepsilon_{\mathbf{k}}$ denotes the single-particle dispersion of the underlying itinerant model and $n_{\mathbf{k}, \sigma}$ is the number operator for particles with momentum $\mathbf{k}$ and spin $\sigma$. In the presence of time reversal symmetry and inversion $\left(n_{\mathbf{k}, \sigma}=n_{-\mathbf{k},-\sigma}\right)$ this can be simplified to

$$
M_{\mathbf{q}}^{1}=\frac{\pi}{N} \sum_{\delta} \sin ^{2}\left(\frac{\mathbf{q} \delta}{2}\right) T_{\delta}
$$

and $\delta$ runs over the vectors connecting a specific lattice site to its respective neighbors with only one representative of the pair $(\delta,-\delta)$. Thus in case of a square lattice $\delta=\hat{\mathbf{x}}, \hat{\mathbf{y}}$ for nearest neighbors, $\delta=\hat{\mathbf{x}}+\hat{\mathbf{y}}, \hat{\mathbf{x}}-\hat{\mathbf{y}}$ for next nearest neighbors etc. and $T_{\delta}$ denotes the kinetic energy in $\delta$-direction. Since $M_{\mathbf{q}}^{1}$ weights more the excitations at higher energies it turns out from Eq. (6) that with increasing $\omega$ the intensity in the transverse magnetic susceptiblity should be confined to wave-vectors around $Q_{A F}=(\pi, \pi)$.

Consider now the case of stripes oriented along the ydirection. Although we are dealing with a perfectly ordered quasi 1D structure we find from our calculations 

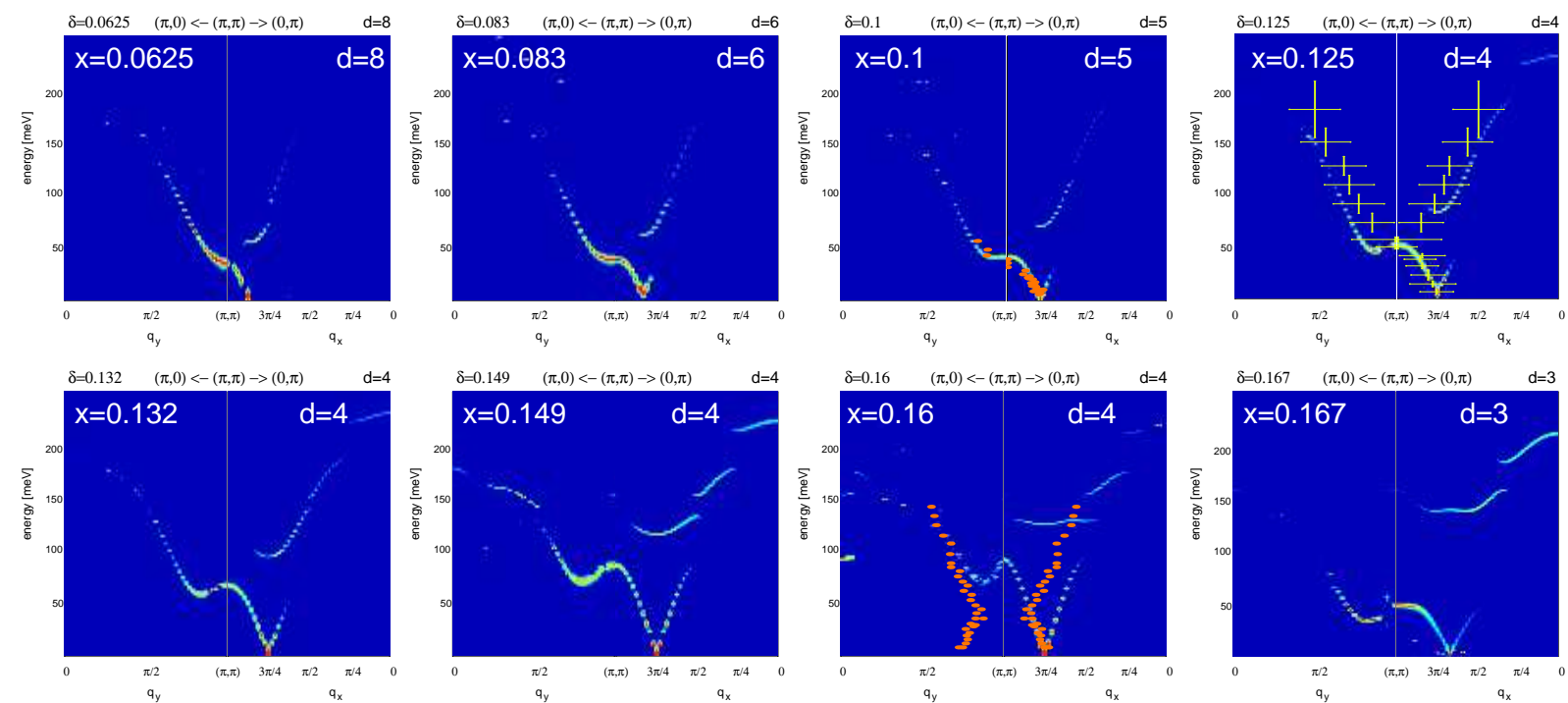

Figure 10: Dispersion of spin excitations perpendicular and along y-axis oriented BC stripes for various dopings. Experimental data are from Ref. [60] $\left(n_{h}=0.1\right)$, Ref. [56] $\left(n_{h}=0.125\right)$, and Ref. [59] $\left(n_{h}=0.16\right)$.

that e.g. for $d=4 \mathrm{BC}$ stripes the kinetic energy along the y-direction is only $\sim 10 \%$ larger than that perpendicular to the stripes. Neglecting the contribution from next-nearest neighbor terms Eq. (6) becomes

$$
M_{\mathbf{q}}^{1}=\frac{\pi T_{\hat{\mathbf{x}}}}{N} \sin ^{2}\left(\frac{q_{x} \delta}{2}\right)+\frac{\pi T_{\hat{\mathbf{y}}}}{N} \sin ^{2}\left(\frac{q_{y} \delta}{2}\right)
$$

so that even in the presence of stripes the high energy magnetic response is essentially two-dimensional but slightly anisotropic around $Q_{A F}$. In Fig. 8 this is exactly the distribution of the optical magnetic excitations one finds above the saddle-point energy. We anticipate that this feature can also be observed in the spin glass-phase of LSCO where due to the imbalance of twin domains the one-dimensional character of low energy spin excitations has been resolved by inelastic neutron scattering experiments [64]. Above $\omega_{s}$ the measurements seem to indicate that the excitations acquire again the (twodimensional) character of the magnons in the undoped system in agreement with the prediction for a striped ground state [29].

Another interesting aspect of the sum rule Eq. 5, that has passed unnoticed so far, is that it allows to measure the change in kinetic energy on entering the superconducting state in a way that is alternative to the proposal in Ref. [63]. The present proposal needs accurate measurements in the higher part of the spectra which should become available with the new pulsed neutron sources.

The overall doping evolution of the vertical stripe magnetic excitations together with availabe experimental data is depicted in Fig. 10. Here we show the exci- tations along scans (a,b) sketched in Fig. 9 It should be noted that the magnetic excitations for SC stripes are almost identical with only slight differences regarding the intensity distribution and the gap structure of optical branches [72]. It turns out from Fig. 10 that for doping $n_{h}>0.125$ the saddle-point excitation $\omega_{s}$ at $Q_{A F}$ rapidly shifts to higher energies together with an increasing softening of the magnetic excitations along the stripe. In fact, the value of $\omega_{s}$ is determined by the magnetic coupling across the domain wall. Because this coupling is mediated by virtual hopping processes of holes between stripe sites and adjacent AF regions it strongly depends on the stripe filling factor $v$ as defined above. As we have shown above for $n_{h}<1 / 8$ the filling of the core of the stripe remains practically constant and $\omega_{s}$ has a weak dependence on doping. On the other hand for $n_{h}>1 / 8$ doping proceeds by changing the filling of the stripes at constant incommensurability. This produces a rapid renormalization of the effective exchange interaction across the core and a concomitant rapid increase in $\omega_{s}$.

In Fig. 11 we compare the doping dependence of $\omega_{s}$ with available experimental data $[56,57,59,60,62,64]$. The latter seem to indicate [59] that in LSCO the saddlepoint excitation at $Q_{A F}$ stays at least constant beyond $n_{h}=1 / 8$ in contrast to what is obtained from the spectra of doped stripes shown in Fig. 10 It is conceivable that instead of doping additional holes into the domain walls these (fluctuating) textures in LSCO stay half-filled beyond $n_{h}=1 / 8$ leading to phase separation between 


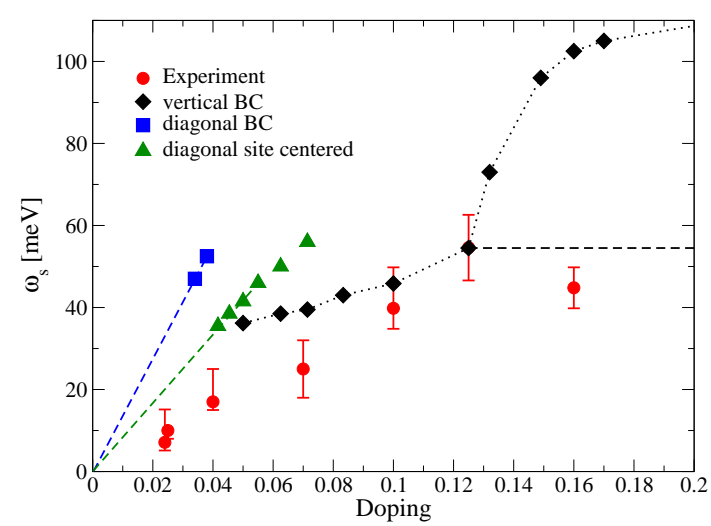

Figure 11: Doping dependence of the saddle-point excitation $\omega_{s}$ at $Q_{A F}$ for diagonal site- and bond-centered stripes and vertical bondcentered stripes. Circles with error bars are experimental data from Refs. [56, 57, 59, 60, 62, 64]. The horizontal dashed line starting from $n_{h}=0.125$ is the expected behavior for a phase separation scenario for stripes.

free carriers and those which are involved in the stripe correlations [25]. On the other hand Wakimoto and collaborators [24] find that at large doping $n_{h}=0.3$ scattering starts around $80 \mathrm{meV}$ which is compatible with a strong increase of $\omega_{s}$ as we predict for the doped stripes. Thus more experimental work is needed at intermediate dopings to clarify the situation.

With underdoping the computations increasingly overestimate the energy of the experimentally determined $\omega_{s}$, especially in the diagonal phase. The main reason for the disagreement in this doping range is probably the increasing disorder character of the stripes. To support this idea we have performed [29] linear spin wave theory calculation of disordered diagonal bond centered stripes. Is is found that already a small amount of disorder leads to a softening and broadening of $\omega_{s}$ which would bring the theoretical data in Fig. 11 closer to the experimental ones.

Recent experimental effort has been made to measure magnetic excitations in LSCO with resonant inelastic $\mathrm{x}$-ray scattering [164]. Because this technique essentially measures in the nuclear Brillouin zone (i.e. close to momentum $\mathbf{q}=0$ ) we show in Fig. 12 the corresponding spectra for $d=4 \mathrm{y}$-axis oriented bond centered stripes. Consider first the scan starting from momentum $\mathbf{q}=(0,0)$ [labeled (d) in Fig. 9] in the direction along the stripe. Because the system is still AF ordered along the stripe (cf. Fig. 2) one has a doubling of the unit cell in this direction. At the same time the doping of the stripe is approximately $v \approx 0.5$ so that the low energy structure of the spectra reflects the continuum of spinon like spin-flip particle-hole excitations for the ac-

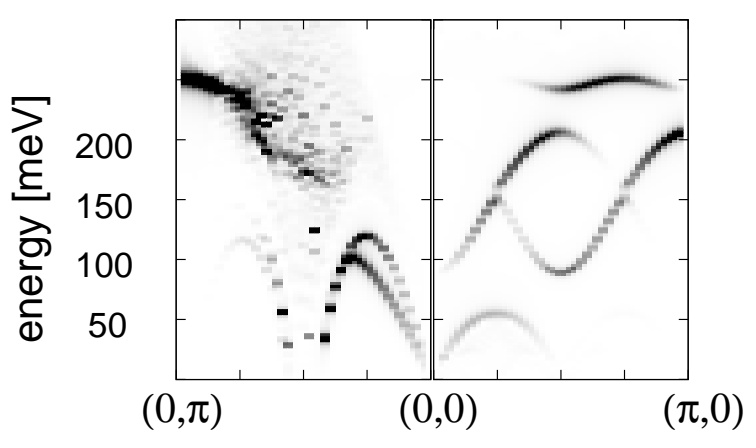

Figure 12: Magnetic excitations in the nuclear Brillouin zone for $d=$ 4 y-oriented stripes. The spectra correspond to the scans labeled (c,d) in Fig. 9

tive band shown in Fig. 6 At higher energy, part of the (high intensity) optical magnon band overlaps with the spin-flip continuum and dominates the spectrum. In the direction perpendicular to the stripe scan (c) is connected to scan (a) by the addition of a reciprocal lattice vector $(3 \pi / 4, \pi)$. Therefore the low energy magnon band which disperses from $(0,0)$ to $(\pi, 0)$ is the replica of the Goldstone mode which has been discussed above. The same holds for the higher energy branches which, however, in the nuclear and magnetic zones strongly differ in weight.

\section{Optical conductivity}

In the previous sections our considerations where mostly based on the extended one-band model, which should be appropriate as long as interband transitions (as in the case of spin excitations) do not play a significant role. In the present section we discuss the optical conductivity of striped systems which involves also higher energy excitations. We therefore investigate the corresponding spectra in the framework of the three-band model which is again treated within the timedependent Gutzwiller approach [25, 26].

It is also possible to obtain an optical conductivity that in the main features resembles experiment, using a one-band Hubbard model. Indeed, one could certainly argue from the inset to Fig. 1b that the transition between LHB and UHB mimics the charge-transfer excitation of cuprates and therefore should provide a reasonable starting point. However, the point is that the charge excitations will also be strongly influenced by the respective stabilitity of bond- and site-centered stripes which are energetically degenerate in the oneband model. This leads to soft lateral fluctuations of the 
stripes (phason mode) which are optically active. Because of the degeneracy between the two textures, the phason is soft in the one-band model at low doping. In contrast, in the more realistic three-band model the two textures become only degenerate at higher doping which, as shown below, is in good agreement with experiment.

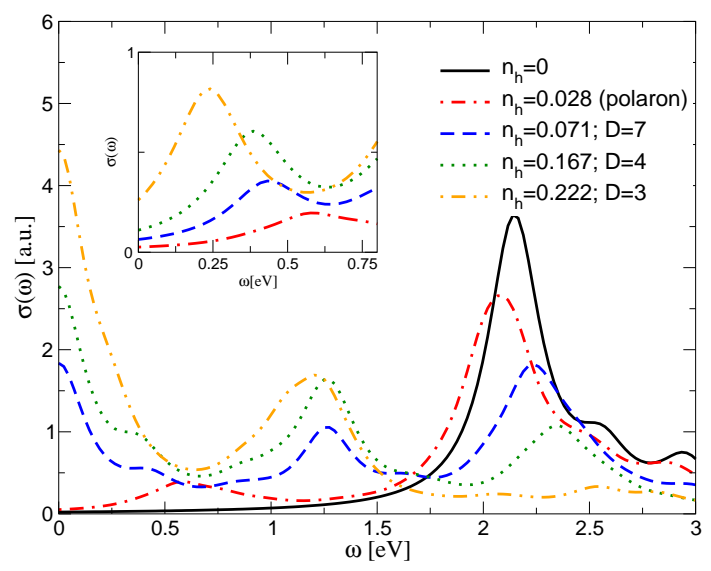

Figure 13: Optical conductivity of the three-band Hubbard model evaluated within the time-dependent GA for various hole concentrations. In the inset: The evolution of the mid-infrared peak as a function of doping. The Drude contribution has set to zero in this case.

Based on the three-band hamiltonian Fig. 13 reports the optical conductivity for AF and inhomogeneous ground states at various dopings. The parameter set has been taken from the first principle computations of Ref. [167] so we have no adjustable parameters.

At very dilute doping $\left(n_{h} \lesssim 0.03\right)$ due to the longrange Coulomb interaction (not included in our calculations) each hole will be close to an acceptor preventing the formation of stripes. The RPA optical conductivity for the corresponding single-hole solution $\left(n_{h}=0.028\right)$ leads to the formation of a doping induced MIR band close to $0.5 \mathrm{eV}$ and doping induced transfer of spectral weight from the charge transfer (CT) band to the MIR region in agreement with experiment in this doping range [151].

For distant stripes $(d=7)$ the single-hole MIR band now splits into two bands. The one at higher energy is a band of incoherent particle-hole excitations close to $1.3 \mathrm{eV}$ which provides a theoretical explanation for the shoulder to the CT excitation seen at the same energy in optical absorption through LSCO thin films [165], and electron energy loss spectroscopy [166].

The low energy MIR peak is a collective mode associated with the soft lateral displacements of the stripe mentioned above. It is located at $0.3 \mathrm{eV}$ at low do- ing and becomes soft only at optimum doping where it merges with the Drude response in good agreement with experiment. This soft collective modes are low energy excitations which have no counterpart in a noninteracting system breaking the paradigm of Fermi liquid theory [26]. It is also possible that coupling to these modes contributes significantly to the pairing. It is worth to remark that new developments in time resolved spectroscopy show [169] that the high energy excitations below the CT band do not participate in the pairing but excitations at the CT band do. The region below $1.6 \mathrm{eV}$ has not been measured yet.

It is interesting to remark that also in nickelates the MIR peak has been attributed to the formation of midgap states due to the stripe ground state [170].

\section{Dynamical stripes}

The investigation of stripe 'dynamical properties' in the previous sections concerned the excitations on top of a stripe ground state with long range order. However, as discussed in Secs.26 in most cuprate materials long range static spin correlations are not observed and also direct evidence for static charge order is quite elusive.

Local probes like STM often point to a broken translational symmetry in real space specially when the high energy part of the spectra is observed. Momentum space probes like ARPES and INS resemble those of an ordered state at high energy but interpolate to a state without broken symmetry at low energy. This points towards a dynamical or a glassy nature of the stripe correlations.

There are two ways in which this dichotomy between low and high energy quasiparticle excitations can be solved: 1) proximity to a quantum/classical critical point in the disordered side (Sec. 8.1). 2) Stripes with long range order but with protected low energy quasiparticles (Sec. 8.2). For the last possibility there are also two variants: 2.a) Long range order in spin and charge and 2.b) Long range order only in the charge.

\subsection{Proximity to a quantum/classical critical point}

The classical version of scenario 1) consists of thermally disordered stripes at finite temperature. This has been the route followed by Vojta and collaborators [78] who have investigated a Landau model for coupled charge and spin fluctuations in the Born-Oppenheimer approximation. Although not mentioned explicitly, this computation also describes a glassy stripe state.

The quantum version of scenario 1) follows from this qualitative argument: In physical dimensions a system may have long (but finite) ranged order parameter spacial correlations which are also long lived close to a 
quantum critical point. This defines a fluctuating frequency $\omega_{0}$ above which the systems appears to be ordered (or at least critical). Then, for energies larger than $\omega_{0}$ with respect to the Fermi level, the spectral function should resemble the spectral function of an ordered system. This high energy spectral weight which normally one would term "incoherent" may in reality carry important information on the momentum structure of the close-by ordered phase. This momentum structure may be determined with ARPES from the momentum distribution $n_{\mathbf{k}}$ by integrating the spectral function over a broad energy window or by STM and INS experiments by direct examination of the high energy spectra.

On the other hand, electrons at lower energies average over the order parameter fluctuations and "sense" a disordered system. In this limit we expect Fermi liquid quasiparticles with all their well known characteristics like a Luttinger Fermi surface. This dichotomy in the momentum structure of low and high energy quasiparticles is consistent with ARPES data [106, 107] on lanthanum cuprates.

In a series of papers [171, 172, 173, 174] which were based on a Kampf-Schrieffer type approach [176] we have worked out the scenario of fermionic quasiparticles coupled to dynamical charge order fluctuations with regard to several experimental observations. These include the angular dependence of the quasiparticle weight in $\mathrm{Bi} 2201$ [177], the isotope shift of the highenergy electronic dispersion [178], and the dichotomy in the Fermi surface of high- $T_{c}$ cuprates [106, 107] as mentioned before.

The Kampf-Schrieffer approach describes quite successfully situations in which the quasiparticles are coupled to locally well formed order-parameter fluctuations but can not be derived from a microscopic approach. On the other hand a formal derivation close to a quantum critical point leads to quasiparticles coupled to diffusive collective modes (CM)s. This leads to a form which is customary in quantum critical phenomena and has often been used for spin fluctuations [179]

$$
D_{\lambda}(\mathbf{q}, \omega)=-\frac{1}{m_{\lambda}+v_{\lambda}\left(\mathbf{q}-\mathbf{q}_{\lambda}\right)^{2}-i \omega-\omega^{2} / \bar{\Omega}_{\lambda}}
$$

where $\lambda=c, s$ refers to charge or spin CMs substantially peaked at characteristic wavevectors $\mathbf{q}_{s} \approx(\pi, \pi)$ and $\mathbf{q}_{c} \approx( \pm \pi / 2,0),(0, \pm \pi / 2)$ respectively. Here the mass $m_{\lambda}$ is the minimum energy required to excite the $\mathrm{CM}$ and $v_{\lambda}$ is a fermion scale setting the CM momentum dispersion. This dispersion is limited by an energy cutoff $\Lambda_{\lambda}$. The dimensionless quantities $m_{\lambda} / \Lambda_{\lambda}$ are the inverse square correlation lengths (in units of the lattice
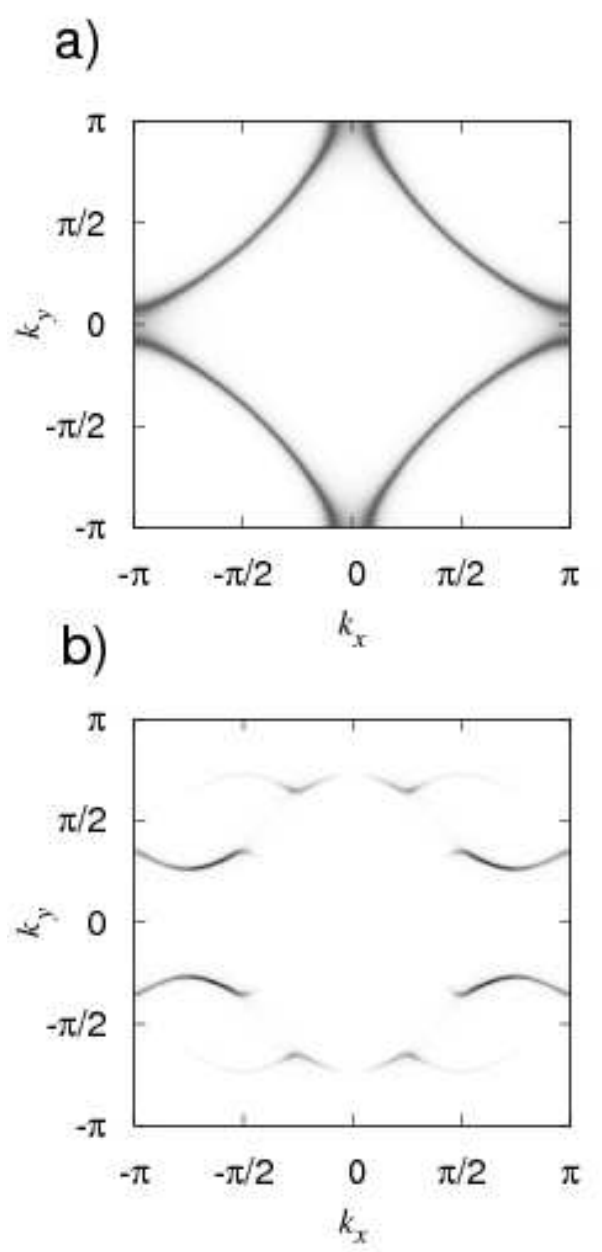

Figure 14: Constant frequency cuts of the electronic dispersion for dynamical $d=4$ bond centered Gutzwiller stripes. (a) $\omega=E_{F}$, (b) $\omega=-0.6 t$. Parameters: $U / t=8, t^{\prime} / t=-0.2$. The frequency structure is obtained from Eq. 12 with $\omega_{0}=0.2 t$ and $v^{2}=0.02$.

spacing), which measure the typical size of ordered domains. The $i \omega$ term establishes the low-energy diffusive character of these fluctuations due to decay into particlehole pairs, whereas above the scale set by $\bar{\Omega}_{\lambda}$ the CM has a more propagating character. This approach has been used to investigate the critical behavior of the lowfrequency optical conductivity [180] and, more recently, to calculate the Raman response function including selfenergy and vertex corrections[181]. In particular, assuming as mediators spin and charge fluctuations with different characteristic wavevectors, it was possible to selectively individuate the charge and spin contributions in the Raman response function. By fitting the experimental spectra in $\mathrm{La}_{2-x} \mathrm{Sr}_{\mathrm{x}} \mathrm{CuO}_{4}$ single crystals within the above theoretical framework, it was found 
that both charge and spin fluctuations were contributing to the quasiparticle scattering (a distinctive feature of stripe fluctuations). However, it was also found that, upon increassing doping, charge CMs progressively acquire more relevance while spin fluctuations become weaker and weaker. This indicates that upon increasing doping into the overdoped regime, stripes naturally evolve into harmonic damped charge-density-wave fluctuations, while spin modes eventually loose weight.

\subsection{Protected low energy quasiparticles}

STM [130, 131] experiments provide strong evidence for the simultaneous existence of low energy (Bogoljubov) quasiparticles and manifestations of truly broken translational symmetry at high energy.

In another recent approach [184, 174] we have developed a phenomenological description with broken translational symmetry but a dynamical order parameter which applies to this situation. The theory also accounts for the contrast reversal in the STM spectra between positive and negative bias [175], in contrast to mean field like static order where this effect in general occurs away from the Fermi level. Here we show that this theory can be combined with the GA computations discussed in Sec. 4 in order to phenomenologically account for the electronic structure of a fluctuating stripe state.

The GA is a renormalized mean-field theory which allows for the definition of an effective Gutzwiller hamiltonian (cf. e.g. Ref. [134])

$$
H^{G A}=\hat{T}+\hat{V} \equiv \sum_{i j \sigma} \widetilde{t_{i j}} c_{i \sigma}^{\dagger} c_{j \sigma}+\sum_{i \sigma} \lambda_{i \sigma} c_{i \sigma}^{\dagger} c_{i \sigma}
$$

where the $c_{i \sigma}^{(\dagger)}$ are now (creation) annihilation operator of the Gutzwiller quasiparticles. The $\widetilde{t_{i j}}$ are renormalized hopping amplitudes and $\lambda_{i \sigma}$ correspond to local chemical potentials. Both parameter sets depend on the value of the onsite repulsion but also on the charge and spin structure of the inhomogeneous ground state.

We want to construct an ansatz for the self energy due to electron-electron interactions. In order to ensure that the self-energy is physical (i.e. that obeys all analytical properties of an electronic self energy) we map the problem to that of electrons coupled to a set of auxiliary states. The idea is to enlarge the Hilbert space by introducing the coupling to an additional set of localized states (described by creation and destruction operators $f_{n \sigma}^{(\dagger)}$ which thus results in the following (auxiliary) Fano-Anderson Hamiltonian,

$$
H^{a u x}=\hat{T}+\sum_{i, n, \sigma} v_{i, n}\left[c_{i \sigma}^{\dagger} f_{i n, \sigma}+\text { h.c. }\right]+\sum_{i, n, \sigma} \varepsilon_{i n} f_{i n, \sigma}^{\dagger} f_{i n, \sigma} .
$$

As a result the Greens function for the Gutzwiller quasiparticles depends on an additional self-energy which is determined by the electronic structure of the $f$-states via

$$
\Sigma_{i}(\omega)=\sum_{n} \frac{v_{i, n}^{2}}{\omega-\epsilon_{i n}^{f}}+\Delta_{i} \equiv f_{i}(\omega)+\Delta_{i} .
$$

with the constant $\Delta_{i}$ controlling the high frequency limit.

Note that this kind of approach automatically maintains the correct analytical properties of the (frequency dependent) self-energy which replaces the static singleparticle potential in Eq. 9 .

For the frequency structure in Eq. 10 we choose a two-pole Ansatz

$$
\begin{aligned}
f_{i}(\omega) & =\left[\frac{\alpha_{i}}{\omega-\omega_{0}}+\frac{\beta_{i}}{\omega+\omega_{0}}\right] \\
\Delta_{i} & =\frac{\alpha_{i}-\beta_{i}}{\omega_{0}}
\end{aligned}
$$

with $\alpha_{i}+\beta_{i}=v^{2}$ and $v^{2}$ controls the strength of the scattering. Thus at each site an asymmetry is introduced in the spectral distribution which below is taken to be proportional to the charge modulation. The constant $\Delta_{i}$ guarantees the limit $\Sigma_{i}(\omega=0)=0$ at each site. Denoting by $\bar{\lambda}$ the average local chemical potential the weights of the poles are choose as $\alpha_{i}=$ $1 / 2\left[1-\tanh \left(1-\lambda_{i} / \bar{\lambda}\right)\right]$.

Fig. 14 reports the corresponding scans of the electronic structure at the Fermi energy (a) and at an energy of $0.6 t$ below $E_{F}$. Clearly the low energy electronic structure resembles that of a homogeneous system due to the fact that the Ansatz Eq. 12 leads to $\Sigma_{i}(\omega=0)=0$. Instead at high energies $\omega \gg \omega_{0}$ the scattering is controlled by $\Sigma_{i}(\omega \rightarrow \infty)=\Delta_{i}$ which according to the above definitions is governed by the local charge and spin structure. One can clearly see the similarities between the electronic structure at $\omega=-0.6 t$ (cf. Fig. $14 \mathrm{~b}$ ) and the reconstructed Fermi surface from the static stripe solution shown in Fig. 7.

Therefore this phenomenological theory reproduces our scenario of low energy protected quasiparticles but emerging spectral properties of charge and spin order at large energies. In contrast to the Kampf-Schrieffer approach [176] which is based on homogeneous ground states the present theory explicitely describes systems with broken symmetry, however, this broken symmetry manifests only at high energies. 


\section{Conclusions}

In this paper we have reviewed evidence for stripe correlations in cuprate superconductors based on Gutzwiller variational calculations supplemented with Gaussian fluctuations. Because our aim is to provide also a quantitative analyis of static and dynamical properties, the appropriate parameter set for lanthanum cuprates based on the extended Hubbard model was derived in Sec. 3 in some detail. Our value of $U / t=8$ agrees with estimates from Ref. [144] and the nextnearest neighbor hopping $t^{\prime} / t=-0.2$ is in the range of values as derived from LDA computations in Ref. [185].

The main emphasis of this review is put on the spin excitations on top of stripe ground states. As we have discussed in Sec. 6 the time-dependent Gutzwiller approach yields an excellent quantitative agreement with the spectra observed by INS when the stripes are static, i.e. in the LBCO compounds. Upon underdoping and in the non-codoped system our calculations in general overestimate the excitation energies. We attribute this discrepancy to the much reduced correlation length as extracted from the low energy spectra due to the dynamics of the stripes and disorder effects [29]. In this regard it would also be interesting to compute the spin excitations on top of the dynamical stripe solutions which have been discussed in Sec. 8

As one approaches the overdoped regime the nature of stripe correlations in the ground state is not so clear since one has to take into account the melting of the stripes and non-Gaussian fluctuation effects will become important. Also it is conceivable that phase separation between the underdoped stripes and an overdoped Fermi liquid occurs as discussed in Ref. [25]. Evidence for this scenario was presented in Sec. 6 from the doping dependence of the saddle point energy. Due to the fact that in the phase separation scenario the population of the domain walls by holes saturates a further increase of the saddle point energy with doping is hampered in agreement with the INS experiments in Ref. [59]. On the other hand, Ref. [24] is in accord with a substantial increase of the saddle point energy.

Another possiblity is that the anharmonic structure of the stripes is gradually lost and stripes become harmonic dynamical and damped charge-density wave fluctuations. This leads to the more standard scenario of a second order transition ending into a quantum critical point slightly above optimal doping[3] driven by a frustrated phase separation mechanism[4]. In this case the combined effect of charge and spin fluctuations can simply be treated within perturbation theory [186].

The natural question which arises is whether the re- sults of Sec. 6 may then also be relevant for an understanding of spin excitations in YBCO. In this compounds the acoustic dispersion shows a low-energy spin gap ( $\Delta \sim 30 \mathrm{meV}$ at optimal doping) and thus does not reach the incommensurate wave-vector at $\omega=0$. Therefore it is more likely that the system is in a quantum disordered spin phase as suggested by the ladder theories [74, 75, 76]. Alternatively a scenario of fluctuating stripes where also the charge loses its long-range order can capture the effect of a spin-gap. [78] One expects that the systems show short range order with a correlation length of the order of $\Delta /(\hbar c) \sim 5 a$ and that for energies larger than $\Delta$ the system resembles that of an ordered phase. For this reason one can expect that a computation as the one discussed in Sec. 6 is suitable for a description of the universal high energy spin response [56, 58, 59, 60, 62, 64, 65, 66, 67, 69]. Again, to capture the excitations over the full energy range would require an approach which incorporates the dynamical nature of stripes as the one proposed in Sec. 8

Concluding, we have shown that the analyis of numerous experiments provides strong evidence for stripe correlations in high- $\mathrm{T}_{c}$ superconductors. This more or less hidden electronic order can account for both normal-state anomalies and high-temperature superconductivity in the cuprates. In fact, the proximity to an instability (particularly if it is a second-order "critical line" ending at zero temperature into a quantum critical point) marking the onset of order naturally brings along abundant fluctuations and leads to strongly temperatureand doping-dependent features, which account for the non-Fermi liquid properties and for a strong pairing interaction. The participation of such stripe fluctuations in the 'pairing glue' is suggested by the recent analysis of Raman scattering data [186] but in any case additional work has to be done in order to elucidate the source of strong scattering/pairing between the charge carriers leading to high- $\mathrm{T}_{c}$.

\section{References}

[1] J. G. Bednorz and K. A. Müller, Z. Physik B 64, 189 (1986).

[2] Proceedings of the Second workshop on phase separation in cuprate superconductors, E. Sigmund and K. A. Müller (eds.); Springer Verlag, Berlin Heidelberg (1994).

[3] C. Castellani, C. Di Castro and M .Grilli, Phys. Rev. Lett. 75, 4650 (1995).

[4] R. Raimondi, et al., Phys. Rev. B 47, 3331 (1993); V. J. Emery and S. Kivelson, Physica C 209, 597 (1993); U. Löw et al., Phys. Rev. Lett. 72, 1918 (1994); Z. Nussinov et al., Phys. Rev. Lett. 83, 472 (1999); J. Lorenzana, C. Castellani, and C. Di Castro, Phys. Rev. B 64, 235127 (2001); J. Lorenzana, C. Castellani, and C. Di Castro, Europhys. Lett. 57, 704 (2002); R. Jamei, S. Kivelson, and B. Spivak, Phys. Rev. Lett. 94, 056805, (2005); C. Ortix, 
J. Lorenzana, M. Beccaria, and C. Di Castro, Phys. Rev. B 75, 195107 (2007); C. Ortix, J. Lorenzana, C. Di Castro, Phys. Rev. Lett. 100. 246402 (2008).

[5] J. Zaanen and O. Gunnarsson, Phys. Rev. B 40, 7391 (1989).

[6] K. Machida, Physica C 158, 192 (1989).

[7] H. J. Schulz, Phys. Rev. Lett. 64, 1445 (1990).

[8] D. Poilblanc and T. M. Rice, Phys. Rev. B 39, 9749 (1989).

[9] S. R. White and D. J. Scalapino, Phys. Rev. Lett. 80, 1272 (1998); S. R. White and D. J. Scalapino, Phys. Rev. Lett. 81, 3227 (1998)

[10] S. R. White and D. J. Scalapino, Phys. Rev. Lett. 91, 136403 , (2003).

[11] S. R. White and D. J. Scalapino, Phys. Rev. B 60, R753 (1999)

[12] C. Stephen Hellberg and E. Manousakis, Phys. Rev. Lett. 83, 132 (1999).

[13] T. Tohyama, C. Gazza, C. T. Shih, Y. C. Chen, T. K. Lee, S. Maekawa, and E. Dagotto, Phys. Rev. B 59, R11649 (1999).

[14] F. Becca, L. Capriotti, and S. Sorella, Phys. Rev. Lett. 87, $167005(2001)$

[15] A. Himeda, T. Kato, and M. Ogata, Phys. Rev. Lett. 88, 117001 (2002).

[16] M. Fleck and A. I. Lichtenstein and E. Pavarini, Phys. Rev. Lett. 84, 4962 (2000); M. Fleck and A. I. Lichtenstein and A. M. Oleś, Phys. Rev. B 64, 134528 (2001).

[17] K. Machida and M. Ichioka, J. Phys. Soc. Jpn. 68, 2168 (1999).

[18] B. Valenzuela, M. A. H. Vozmediano, and F. Guinea, Phys. Rev. B 62, 11312 (2000).

[19] B. Normand and A. P. Kampf, Phys. Rev. B 65, 020509 (2001).

[20] M. G. Zacher, R. Eder, E. Arrigoni, and W. Hanke, Phys. Rev. Lett. 85, 824 (2000).

[21] V. I. Anisimov, M. A. Korotin, A. S. Mylnikova, A. V. Kozhevnikov, Dm. M. Korotin, and J. Lorenzana, Phys. Rev. B 70, 17250 (2004).

[22] G. Seibold, C. Castellani, C. Di Castro, and M. Grilli, Phys. Rev. B 58, 13506 (1998).

[23] G. Seibold, Phys. Rev. 58, 15520 (1998).

[24] S. Wakimoto, K. Yamada, J. M. Tranquada, C. D. Frost, R. J. Birgeneau, and H. Zhang, Phys. Rev. Lett. 98, 247003 (2007)

[25] J. Lorenzana and G. Seibold, Phys. Rev. Lett. 89, 136401 (2002).

[26] J. Lorenzana and G. Seibold, Phys. Rev. Lett. 90, 066404 (2003).

[27] G. Seibold and J. Lorenzana, Phys. Rev. B 69, 134513 (2004).

[28] G. Seibold, J. Lorenzana, and M. Grilli, Phys. Rev. B 75, 100505 (2007)

[29] G. Seibold and J. Lorenzana, Phys. Rev. B 80, 012509 (2009).

[30] G. Seibold, R. S. Markiewicz, and J. Lorenzana, Phys. Rev. B 83, 205108 (2011)

[31] J. M. Tranquada, B. J. Sternlieb, J. D. Axe, Y. Nakamura and S. Uchida, Nature 375, 56 (1995)

[32] J. M. Tranquada, J. D. Axe, N. Ichikawa, Y. Nakamura, S. Uchida, and B. Nachumi, Phys. Rev. B 54, 7489 (1996).

[33] J. M. Tranquada, J. D. Axe, N. Ichikawa, A. R. Moodenbaugh, Y. Nakamura, and S. Uchida, Phys. Rev. Lett. 78, 338 (1997).

[34] S. M. Hayden, G. H. Lander, J. Zarestky, P. J. Brown, C. Stassis, P. Metcalf, and J. M. Honig, Phys. Rev. Lett. 68, 1061 (1992).

[35] C. H. Chen, S-W. Cheong and A. S. Cooper, Phys. Rev. Lett. 71, 2461 (1993).

[36] J. M. Tranquada, D. J. Buttrey, V. Sachan, and J. E. Lorenzo, Phys. Rev. Lett. 73, 1003 (1994)

[37] M. Fujita, H. Goka, K. Yamada, and M. Matsuda, Phys. Rev. Lett. 88, 167008 (2002).

[38] Klauss, H.-H., Wagener, W., Hillberg, M., Kopmann, W., Walf, H., Litterst, F. J., Hücker, M. and Büchner, B., Phys. Rev. Lett. 85, 4590 (2000).

[39] M. Hücker, M. v. Zimmermann, M. Debessai, J. S. Schilling,
J. M. Tranquada, and G. D. Gu, Phys. Rev. Lett. 104, 057004 (2010).

[40] M. Fujita, M. Enoki, S. Iikubo, K. Kudo, N. Kobayashi, and K. Yamada, arXiv:0903.5391

[41] M. v. Zimmermann, A. Vigliante, T. Niemöller, N. Ichikawa, T. Frello, J. Madsen, P. Wochner, S. Uchida, N. H. Andersen, J. M. Tranquada, D. Gibbs and J. R. Schneider, EPL 41, 629 (1998).

[42] P. Abbamonte, A. Rusydi, S. Smadici, G. D. Gu, G. A. Sawatzky, and D. L. Feng, Nature Phys. 1, 155 (2005).

[43] J. Fink, E. Schierle, E. Weschke, J.Geck, D. Hawthorn, V. Soltwisch, H. Wadati, and Hsueh-Hung Wu, Phys. Rev. B 79, 100502 (2009).

[44] K. Yamada, C. H. Lee, K. Kurahashi, J. Wada, S. Wakimoto, S. Ueki, H. Kimura, Y. Endoh, S. Hosoya, G. Shirane, R. J. Birgeneau, M. Greven, M. A. Kastner, and Y. J. Kim, Phys. Rev. B 57, 6165 (1998).

[45] S. Wakimoto, H. Zhang, K. Yamada, I. Swainson, Hyunkyung Kim, and R. J. Birgeneau, Phys. Rev. Lett. 92, 217004 (2004).

[46] S. Wakimoto, G. Shirane, Y. Endoh, K. Hirota, S. Ueki, K. Yamada, R. J. Birgeneau, M. A. Kastner, Y. S. Lee, P. M. Gehring and S. H. Lee, Phys. Rev. B 60, R769 (1999).

[47] S. Wakimoto, R. J. Birgeneau, M. A. Kastner, Y. S. Lee, R. Erwin, P. M. Gehring, S. H. Lee, M. Fujita, K. Yamada, Y. Endoh, K. Hirota, and G. Shirane, Phys. Rev. B 61, 3699 (2000).

[48] M. Matsuda, M. Fujita, K. Yamada, R. J. Birgeneau, M. A. Kastner, H. Hiraka, Y. Endoh, S. Wakimoto, and G. Shirane, Phys. Rev. B 62, 9148 (2000).

[49] M. Fujita, K. Yamada, H. Hiraka, P. M. Gehring, S. H. Lee, S. Wakimoto and G. Shirane, Phys. Rev. B 65, 064505 (2002).

[50] M. Fujita, H. Goka, K. Yamada, J. M. Tranquada, and L. P. Regnault, Phys. Rev. B 70, 104517 (2004).

[51] M. Matsuda, M. Fujita, K. Yamada, R. J. Birgeneau, Y. Endoh, and G. Shirane, Phys. Rev. B 65, 134515 (2002).

[52] M. Arai, , T. Nishijima, Y. Endoh, T. Egami, S. Tajima, K. Tomimoto, Y. Shiohara, M. Takahashi, A. Garrett, and S. M. Bennington, Phys. Rev. Lett. 83, 608 (1999).

[53] P. Dai, H. A. Mook, R. D. Hunt, and F. Dogan, Phys. Rev. B 63, 054525 (2001).

[54] D. Haug, V. Hinkov, Y. Sidis, P. Bourges, N. B. Christensen, A. Ivanov, T Keller, C. T. Lin and B. Keimer, New J. Phys. 12, 105006 (2010).

[55] In some measurements only an approach of incommensurate branches towards $\mathrm{Q}_{A F}$ is resolved with a subsequent dispersion outwards with increasing energy.

[56] J. M. Tranquada, H. Woo, T. G. Perring, H. Goka, G. D. Gu, G. $\mathrm{Xu}, \mathrm{M}$. Fujita, and K. Yamada, Nature (London) 429, 534 (2004).

[57] H. Hiraka et al., J. Phys. Soc. Jpn. 70, 853 (2001).

[58] N. B. Christensen, D. F. McMorrow, H. M. Rønnow, B. Lake, S. M. Hayden, G. Aeppli, T. G. Perring, M. Mangkorntong, M. Nohara, and H. Tagaki, Phys. Rev. Lett. 93, 147002 (2004).

[59] B. Vignolle, S. M. Hayden, D. F. McMorrow, H. M. Ronnow, C.D. Frost, and T. G. Perring, Nat. Phys. 3, 163 (2007).

[60] M. Kofu, T. Yokoo, F. Trouw, and K. Yamada,arXiV:0710:5766.

[61] V. Hinkov, S. Pailhés, P. Bourges, Y. Sidis, A. Ivanov, A. Kulakov, C. Lin, D. Chen, C. Bernhard, and B. Keimer, Nature (London) 430, 650 (2004).

[62] M. Matsuda, M. Fujita, S. Wakimoto, J. A. Fernandez-Baca, J. M. Tranquada, and K. Yamada, Phys. Rev. Lett. 101, 197001 (2008).

[63] D. J. Scalapino and S. R. White, Phys. Rev. B 58, 8222 (1998).

[64] M. Matsuda, J. A. Fernandez-Baca, M. Fujita, K. Yamada, and J. M. Tranquada, Phys. Rev. B 84, 104524 (2011).

[65] S. M. Hayden, H. A. Mook, Pengcheng Dai, T. G. Perring and F. Dogan, Nature 429, 531 (2004).

[66] D. Reznik, P. Bourges, L. Pintschovius, Y. Endoh, Y. Sidis, T. 
Masui, and S. Tajima, Phys. Rev. Lett. 93, 207003 (2004).

[67] C. Stock, W. J. L. Buyers, R. A. Cowley, P. S. Clegg, R. Coldea, C. D. Frost, R. Liang, D. Peets, D. Bonn, W. N. Hardy, and R. J. Birgeneau, Phys. Rev. B 71, 024522 (2005).

[68] Hinkov, P. Bourges, S. Pailhés, Y. Sidis, A. Ivanov, C. D. Frost, T. G. Perring, C. T. Lin, D. P. Chen, and B. Keimer, Nat. Phys. 3 , 780 (2007).

[69] V. Hinkov, D. Haug, B. Fauqué, P. Bourges, Y. Sidis, A. Ivanov, C. Bernhard, C. T. Lin and B. Keimer, Science 319 (2008)

[70] B. Fauqué, Y. Sidis, L. Capogna, A. Ivanov, K. Hradil, C. Ulrich, A. I. Rykov, B. Keimer, and P. Bourges, Phys. Rev. B 76, 214512 (2007)

[71] Guangyong Xu, G. D. Gu, M. Hücker, B. Fauqué, T. G. Perring, L. P. Regnault, and J. M. Tranquada, Nat. Phys. 5, 642 (2009).

[72] G. Seibold and J. Lorenzana, Phys. Rev. Lett. 94, 107006 (2005).

[73] G. Seibold and J. Lorenzana, Phys. Rev. B73, 144515 (2006)

[74] M. Vojta and T. Ulbricht, Phys. Rev. Lett. 93, 127002 (2004).

[75] G. S. Uhrig, K. P. Schmidt, and M. Grüninger, Phys. Rev. Lett. 93, 267003 (2004).

[76] G. S. Uhrig, K. P. Schmidt, and M. Grüninger, J.Phys.Soc.Jpn. 74 (2005), Supplement 86.

[77] B. M. Andersen and P. Hedegård, Phys. Rev. Lett. 95, 037002 (2005).

[78] M. Vojta, T. Vojta, and R. K. Kaul, Phys. Rev. Lett. 97, 097001 (2007).

[79] Brian M. Andersen and Olav F. Syljuåsen, Phys. Rev. B 75 , 012506 (2007)

[80] Brian M. Andersen, Siegfried Graser, and P. J. Hirschfeld, Phys. Rev. Lett. 105, 147002 (2010)

[81] F. Krüger and S. Scheidl, Phys. Rev. B 67, 134512 (2003).

[82] E. W. Carlson, D. X. Yao, and D. K. Campbell, Phys. Rev. B 70, 064505 (2004)

[83] D. X. Yao, E. W. Carlson, and D. K. Campbell, Phys. Rev. Lett. 97, 017003 (2006)

[84] A. T. Boothroyd, P. Babkevich, D. Prabhakaran, and P. G. Freeman, Nature 471, 341 (2011).

[85] S. Krämer and M. Mehring, Phys. Rev. Lett. 83, 396 (1999).

[86] G. B. Teitel'baum, B. Büchner, and H. de Gronckel, Phys. Rev. Lett. 84, 2949 (2000).

[87] P. M. Singer, A. W. Hunt, and T. Imai, Phys. Rev. Lett. 88, 047602 (2002)

[88] J. Haase, C. P. Slichter, and C. T. Milling, J. Supercond. 15, 339 (2002).

[89] T. Wu, H. Mayaffre, S. Kramer, M. Horvatic, C. Berthier, W.N. Hardy, R. Liang, D.A. Bonn, and M.-H. Julien, Nature 477, 191 (2011).

[90] M. Vojta, Adv. Phys. 58, 699 (2009)

[91] R. Daou, J. Chang, D. LeBoeuf, O. Cyr-Choiniere, F. Laliberte, N. Doiron-Leyraud, B. J. Ramshaw, R. Liang, D. A. Bonn, W. N. Hardy, and L. Taillefer, Nature 463, 519 (2010).

[92] , Jan Brinckmann and Patrick A. Lee, Phys. Rev. Lett. 82, 2915 (1999).

[93] M. R. Norman,Phys. Rev. B 61, 14751 (2000).

[94] Andrey V. Chubukov, Boldizsár Jankó, and Oleg Tchernyshyov, Phys. Rev. B 63, 180507(R) (2001)

[95] A. P. Schnyder, A. Bill, C. Mudry, R. Gilardi, H. M. Ronnow, and J. Mesot, Phys. Rev. B 70, 214511 (2004).

[96] M. Berciu and S. John, Phys. Rev. B 69, 224515 (2004).

[97] A. Sherman and M. Schreiber, Phys. Rev. B 69, 100505 (2004).

[98] I. Eremin, D. K. Morr, A. V. Chubukov, K. H. Bennemann, and M. R. Norman, Phys. Rev. Lett. 94, 147001 (2005).

[99] A. Sherman, arXiv:1108.4179

[100] Hiroyuki Yamase and Walter Metzner, Phys. Rev. B 73, 214517 (2006)
[101] D. Reznik, Adv. Cond. Matt. Phys. 2010, Article ID 523549.

[102] E. Kaneshita, M. Ichioka, and K.Machida, Phys. Rev. Lett. 88, 115501 (2002).

[103] S. I. Mukhin, A. Mesaros, J. Zaanen, and F. V. Kusmartsev, Phys. Rev. B 76, 174521 (2007).

[104] M.I. Salkola, V.J. Emery, and S.A. Kivelson, J. Supercond. 9, 401 (1996).

[105] G. Seibold, F. Becca, F. Bucci, C. Castellani, C. Di Castro, M. Grilli, Eur. Phys. J B 13, 87 (2000).

[106] X. J. Zhou, P. Bogdanov, S. Kellar, T. Noda, H. Eisaki, S. Uchida, Z. Hussain, and Z.-X. Shen, Science 286, 268 (1999).

[107] X. J. Zhou, T. Yoshida, S. A. Kellar, P. V. Bogdanov, E. D. Lu, A. Lanzara, M. Nakamura, T. Noda, T. Kakeshita, H. Eisaki, S. Uchida, A. Fujimori, Z. Hussain, and Z. X. Shen, Phys. Rev. Lett. 86, 5578 (2001)

[108] A. Ino, T. Mizokawa, A. Fujimori, K. Tamasaku, H. Eisaki, S. Uchida, T. Kimura, T. Sasagawa, and K. Kishio, Phys. Rev. Lett. 79, 2101 (1997)

[109] N. Harima, J. Matsuno, A. Fujimori, Y. Onose, Y. Taguchi, and Y. Tokura, Phys. Rev. B 64, R220507 (2001).

[110] N. Doiron-Leyraud, C. Proust, D. LeBoeuf, J. Levallois, J.B. Bonnemaison, R. Liang, D. A. Bonn, W. N. Hardy, and L. Taillefer, Nature 447, 565 (2007).

[111] E. A. Yelland, J. Singleton, C. H. Mielke, N. Harrison, F. F. Balakirev, B. Dabrowski, and J. R. Cooper, arXiv:0707.0057

[112] A. F. Bangura, J. D. Fletcher, A. Carrington, J. Levallois, M. Nardone, B. Vignolle, P. J. Heard, N. Doiron-Leyraud, D. LeBoeuf, L. Taillefer, S. Adachi, C. Proust, and N. E. Hussey, arXiv:0707.4461

[113] D. LeBoeuf, N. Doiron-Leyraud, R. Daou, J.-B. Bonnemaison, J. Levallois, N. E. Hussey, C. Proust, L. Balicas, B. Ramshaw, R. Liang, D. A. Bonn, W. N. Hardy, S. Adachi, and L. Taillefer, Nature 450, 533 (2007).

[114] A. J. Millis and M. R. Norman, Phys. Rev. B 76, 220503(R) (2007).

[115] F. Laliberte, J. Chang, N. Doiron-Leyraud, E. Hassinger, R. Daou, M. Rondeau, B. J. Ramshaw, R. Liang, D. A. Bonn, W. N. Hardy, S. Pyon, T. Takayama, H. Takagi, I. Sheikin, L. Malone, C. Proust, K. Behnia, L. Taillefer, Nature Communications 2, 432 (2011).

[116] Wang, Y., Li, P., and Ong, N. P., Phys. Rev. B 73, 024510 (2006).

[117] Olivier Cyr-Choinire, R. Daou, Francis Laliberté, David LeBoeuf, Nicolas Doiron-Leyraud, J. Chang, J.-Q. Yan, J.-G. Cheng, J.-S. Zhou, J. B. Goodenough, S. Pyon, T. Takayama, H. Takagi, Y. Tanaka, and Louis Taillefer, Nature 458, 743 (2009).

[118] Christian Hess, Emad M. Ahmed, Udo Ammerahl, Alexandre Revcolevschi, Bernd Büchner, Eur. Phys. J. Special Topics 188, 103 (2010).

[119] Ivar Martin and C. Panagopoulos, Eur. Phys. Lett. 91, 67001 (2010).

[120] Andreas Hackl and Matthias Vojta, New J. Phys. 12, 105011 (2010).

[121] J. E. Hoffmann, K. McElroy, D.-H. Lee, K. M Lang, H. Eisaki, S. Uchida and J. C. Davis, Science 297, 1148 (2002).

[122] C. Howald, H. Eisaki, N. Kaneko, M. Greven, and A. Kapitulnik, Phys. Rev. B 67, 014533 (2003).

[123] K. McElroy, D.-H. Lee, J. E. Hoffman, K. M. Lang, J. Lee, E. W. Hudson, H. Eisaki, S. Uchida, and J. C. Davis, Phys. Rev. Lett. 94, 197005 (2005).

[124] A. Hashimoto, N. Momono, M. Oda, and M. Ido, Phys. Rev. B 74, 064508 (2006).

[125] T. Hanaguri, Y. Kohsaka, J. C. Davis, C. Lupien, I. Yamada, M. Azuma, M. Takano, K. Ohishi, M. Ono, and H. Takagi, Nature Physics 3, 865 (2007) 
[126] W. D. Wise, M. C. Boyer, Kamalesh Chatterjee, Takeshi Kondo, T. Takeuchi, H. Ikuta, Yayu Wang, and E. W. Hudson, Nature Physics 4, 696 (2008).

[127] M. Vershinin, Shashank Misra1, S. Ono, Y. Abe2, Yoichi Ando and Ali Yazdani, Science 303, 1995 (2004).

[128] T. Hanaguri, C. Lupien, Y. Kohsaka, D.-H. Lee, M. Azuma, M. Takano, H. Takagi, and J. C. Davis, Nature 430, 1001 (2004).

[129] Q. H. Wang and D.-H. Lee, Phys. Rev. B 67, 020511(R) (2003)

[130] Y. Kohsaka, C. Taylor, P. Wahl, A. Schmidt, Jhinhwan Lee, K. Fujita, J. W. Alldredge, K. McElroy, Jinho Lee, H. Eisaki, S. Uchida, D.-H. Lee, and J. C. Davis, Nature 454, 1072 (2008).

[131] J. W. Alldredge, Jinho Lee, K. McElroy, M. Wang, K. Fujita, Y. Kohsaka, C. Taylor, H. Eisaki, S. Uchida, P. J. Hirschfeld, and J. C. Davis, Nature Physics 4, 319 (2008).

[132] M. C. Gutzwiller, Phys. Rev. 137, A1726 (1965).

[133] G. Seibold and J. Lorenzana, Phys. Rev. Lett. 86, 2605 (2001).

[134] G. Seibold, F. Becca, and J. Lorenzana, Phys. Rev. B 67, 085108 (2003)

[135] G. Seibold, F. Becca, P. Rubin, and J. Lorenzana, Phys. Rev. B 69, 155113 (2004)

[136] G. Seibold, F. Becca, and J. Lorenzana, Phys. Rev. Lett. 100, 016405 (2008); G. Seibold, F. Becca, and J. Lorenzana, Phys Rev. B 78, 045114 (2008).

[137] M. Ichioka, E. Kaneshita, and K. Machida, J. Phys. Soc. Jpn. 70, 818 (2001)

[138] E. Kaneshita, M Ichioka, and K. Machida J. Phys. Soc. Jpn, 70, 866 (2001).

[139] E. Kaneshita, R. Morino, M. Ichioka, and K. Machida, J. Phys. Chem. Solids 63, 1545 (2002).

[140] E. Kaneshita, M. Ichioka, and K. Machida, Phys. Rev. Lett. 88, 115501 (2002)

[141] S. Varlamov and G. Seibold, Phys. Rev. B 65, 075109 (2002)

[142] M. Inui and P. B. Littlewood, Phys. Rev. B 44, 4415 (1991).

[143] R. R. P. Singh and M. P. Gelfand, Phys. Rev. B 52, R15695 (1995).

[144] R. Coldea, S. M. Hayden, G. Aeppli, T. G. Perring, C. D. Frost, T. E. Mason, S.-W. Cheong, and Z. Fisk, Phys. Rev. Lett. 86, 5377 (2001)

[145] N. S. Headings, S. M. Hayden, R. Coldea, and T. G. Perring Phys. Rev. Lett. 105, 247001 (2010).

[146] M. Roger and J. M. Delrieu, Phys. Rev. B 39, 2299 (1989).

[147] H. J. Schmidt and Y. Kuramoto, Physica C 167, 263 (1990).

[148] F. Lema, J. M. Eroles, C. D. Batista, and E. Gagliano, Phys Rev. B 55, 15295 (1997).

[149] J. Lorenzana, J. Eroles, and S. Sorella, Phys. Rev. Lett. 83, 5122 (1999).

[150] Weihong Zheng, Rajiv R. P. Singh, Jaan Oitmaa, Oleg P. Sushkov, and Chris J. Hamer, Phys. Rev. B 72, 033107 (2005).

[151] S. Uchida, T. Ido, H. Takagi, T. Arima, Y. Tokura, and S Tajima, Phys. Rev. B 43, 7942 (1991).

[152] H. Okamoto, T. Miyagoe, K. Kobayashi, H. Uemura, H. Nishioka, H. Matsuzaki, A. Sawa, and Y. Tokura, Phys. Rev. B 83, 125102 (2011).

[153] Strictly speaking this coincidence only shows up for $n_{h} \gtrsim 0.1$ and susceptibilities evaluated with electronic structure parameters appropriate for LSCO.

[154] R. S. Markiewicz, J. Lorenzana, and G. Seibold, Phys. Rev. B 81, 014510 (2010).

[155] M. Granath, Phys. Rev. B 69, 214433 (2004).

[156] H. Eskes, L. H. Tjeng, G. A. Sawatzky, Phys. Rev. B 42, 288 (1990)

[157] Y. Kohsaka, C. Taylor, K. Fujita, A. Schmidt, C. Lupien, T. Hanaguri, M. Azuma, M. Takano, H. Eisaki, H. Takagi, S Uchida, and J. C. Davis, Science 315, 1380 (2007).
[158] J. Lorenzana, G. Seibold, and R. Coldea, Phys. Rev. B 72 224511 (2005).

[159] B. Normand and A. P. Kampf, Phys. Rev. B 64, 024521 (2001).

[160] Rui-Hua He, M. Fujita, M. Enoki, M. Hashimoto, S. Iikubo, S.K. Mo, Hong Yao, T. Adachi, Y. Koike, Z. Hussain, Z.-X. Shen, and K. Yamada, Phys. Rev. Lett. 107, 127002 (2011).

[161] M. Raczkowski, R. Frésard, and A. M. Oleś, Europhysics Letters 76, 128 (2006).

[162] M. Raczkowski, R. Frésard, and A. M. Oleś, Phys. Stat. Sol. (b) 244, 2521 (2007).

[163] G. B. Martins, C. Gazza, J. C. Xavier, A. Feiguin, and E. Dagotto, Phys. Rev. Lett. 84, 5844 (2000).

[164] L. Braicovich, J. van den Brink, V. Bisogni, M. Moretti Sala, L. J. P. Ament, N. B. Brookes, G. M. De Luca, M. Salluzzo, T. Schmitt, V. N. Strocov, and G. Ghiringhelli, Phys. Rev. Lett. 104, 077002 (2010).

[165] M. Suzuki, Phys. Rev. B 39, 2312 (1989). P

[166] M. Terauchi and M. Tanaka, Micron 30, 371 (1999).

[167] A. K. McMahan, J. F. Annett, and R. M. Martin, Phys. Rev. B 42, 6268 (1990).

[168] M. S. Hybertsen, E. B. Stechel, W. M. C. Foulkes, and M. Schlüter, Phys. Rev. B 45, 10032 (1992).

[169] B. Mansart, J. Lorenzana, M. Scarongella, M. Chergui and F. Carbone, arXiv:1112.0737

[170] C. C. Homes, J. M. Tranquada*, Q. Li, A. R. Moodenbaugh, D. J. Buttrey, Phys. Rev. B 67, 184516 (2003).

[171] G. Seibold and M. Grilli, Phys. Rev. B 63, 224505 (2001).

[172] G. Seibold and M. Grilli, Phys. Rev B 72, 104519 (2005).

[173] M. Grilli, G. Seibold, A. Di Ciolo, and J. Lorenzana, Phys. Rev. B 79, 125111 (2009)

[174] G. Seibold, M. Grilli, and J. Lorenzana, New Journal of Physics 12, 105010 (2010).

[175] J.-H. Ma, Z.-H. Pan, F. C. Niestemski, M. Neupane, Y.-M. Xu, P. Richard, K. Nakayama, T. Sato, T. Takahashi, H.-Q. Luo, L. Fang, H.-H. Wen, Ziqiang Wang, H. Ding, and V. Madhavan, Phys. Rev. Lett. 101, 207002 (2008).

[176] A. P. Kampf and J. R. Schrieffer, Phys. Rev. B42, 7967 (1990).

[177] Takeshi Kondo, Rustem Khasanov, Tsunehiro Takeuchi, Jörg Schmalian and Adam Kaminski, Nature 457, 296 (2009).

[178] G.-H. Gweon, T. Sasagawa, S.Y. Zhou, J. Graf, H. Takagi, D.H. Lee, and A. Lanzara, Nature 430, 187 (2004).

[179] Ar. Abanov, A. Chubukov, and J. Schmalian, Adv. Phys. 52, 119 (2003), and references therein

[180] S. Caprara, M. Grilli, C. Di Castro, and T. Enss, Phys. Rev. B 75, 140505 (2007).

[181] S. Caprara, C. Di Castro, B. Muschler, W. Prestel, R. Hackl, M. Lambacher, A. Erb, S. Komiya, Y. Ando, and M. Grilli, Phys. Rev. B 84, 0504508 (2011).

[182] A. Bianconi, N. L. Saini, A. Lanzara, M. Missori, T. Rossetti, H. Oyanagi, H. Yamaguchi, K. Oka, and T. Ito, Phys. Rev. Lett. 76, 34123415 (1996).

[183] M. Fratini, N. Poccia, A. Ricci, G. Campi, M. Burghammer, G. Aeppli, and A. Bianconi, Nature 466, 841 (2010).

[184] G. Seibold, M. Grilli, and J. Lorenzana, Phys. Rev. Lett. 103, 217005 (2009).

[185] E. Pavarini, I. Dasgupta, T. Saha-Dasgupta, O. Jepsen, and O. K. Andersen Phys. Rev. Lett. 87, 047003 (2001).

[186] S. Caprara, C. Di Castro, B. Muschler, W. Prestel, R. Hackl, M. Lambacher, A. Erb, S. Komiya, Y. Ando, and M. Grilli, Phys. Rev. B 84, 054508 (2011). 